\title{
Modelización y análisis de escenarios de intervención en sistemas socio-naturales: El caso del sistema de sustentabilidad energía-territorio de la región de Coquimbo, Chile
}

\author{
Modeling and analysis of intervention scenarios in socio-natural systems: The case \\ of energy-territory sustainability system of the Coquimbo Region, Chile
}

\author{
Rodrigo Ramos-Jiliberto ${ }^{1}$, Rodrigo Jiliberto Herrera ${ }^{2}$
}

[Recibido: 18 de marzo 2020, Aceptado: 27 de agosto 2020, Corregido: 18 de setiembre 2020, Publicado: 1 de enero 2021]

\section{Resumen}

[Introducción]: Este articulo propone avances en la modelización y el análisis de los sistemas socio-naturales objeto de las políticas públicas en medio ambiente y desarrollo sustentable. [Objetivo]: Presentar una propuesta de modelización sistémica formal de sistemas socio-naturales en contextos de decisión estratégicos. [Metodología]: Basados en el lenguaje de los digrafos signados, presentamos un conjunto de elementos de sistemas socio-naturales modelizados, orientados al análisis prospectivo de la aplicación de diversos escenarios de intervención. Mostramos también un conjunto de nuevas métricas cuantitativas para evaluar el rol dinámico de las variables componentes del sistema socio-natural. Se toma como caso de estudio el sistema de sustentabilidad de energía en el territorio de la región de Coquimbo, Chile, y se evalúa comparativamente el desempeño esperado de medidas de gestión ambiental. [Resultados]: Los resultados de este estudio sugieren que las medidas de intervención/gestión planificadas en función de las nuevas métricas presentadas en este artículo, resultan eficaces de acuerdo con los escenarios proyectados por la modelización. [Conclusiones]: La modelización estructural cualitativa es adecuada para representar y analizar sistemas socio-naturales, lo que exhibe ventajas operativas para apoyar la toma de decisiones.

Palabras clave: Digrafos signados; modelización cualitativa; sistemas socio-ecológicos; teoría de grafos; teoría de sistemas.

\begin{abstract}
[Introduction]: This study proposes advances in modeling and analysis of socio-natural systems as a result of public policies on the environment and sustainable development. [Objective]: Present a formal systemic proposal for modeling socio-natural systems in a strategic decision-making framework. [Methodology]: Based on the symbols of signed digraphs, the study offers a group of elements to analyze socio-natural model systems geared towards a prospective analysis of diverse intervention scenarios applied to the systems. New quantitative metrics are also presented to evaluate the dynamic role of the variables comprising the socio-natural systems. The energy-territory

1 Investigador del Centro Genómica, Ecología \& Medio Ambiente (GEMA), Facultad de Estudios Interdisciplinarios, Universidad Mayor, Chile; rodrigo.ramos@umayor.cl, https://orcid.org/0000-0002-0108-7502

2 Profesor de Estudios Transversales en Humanidades para las Ingenierías y Ciencias (ETHICS) de la Facultad de Ciencias Físicas y Matemáticas, Universidad de Chile, Chile; rodrigo.jiliberto@uchile.cl, https://orcid.org/0000-0002-7336-8223
\end{abstract}

(cc)(P)(




\section{Revista de CIENCIAS AMBIENTALES Tropical Journal of Environmental Sciences}

Revista de Ciencias Ambientales (Trop J Environ Sci) e-ISSN: 2215-3896 (Enero-Junio, 2021) . Vol 55(1): 1-31 DOI: https://doi.org/10.15359/rca.55-1.1

Open Access: www.revistas.una.ac.cr/ambientales e-mail: revista.ambientales@una.ac.cr Ramos-Jiliberto R., Jiliberto-Herrera R.

sustainability system of the Coquimbo Region in Chile is used as a case study, and the expected performance of alternative environmental management actions is evaluated on a comparative basis. [Results]: Results suggest that intervention/management actions planned based on the new metrics presented here were particularly effective in the projected modeling scenarios. [Conclusions]: Qualitative structural modeling is suitable for representing and analyzing socio-natural systems, exhibiting operational advantages to support decision-making.

Keywords: Graph theory; qualitative modeling; signed digraphs; social-ecological systems; systems theory.

\section{Introducción}

Los sistemas socio-naturales, también llamados sistemas socio-ecológicos, son entidades integradas y persistentes compuestas por elementos sociales (p.ej., económicos, políticos, urbanos, industriales) y naturales (p.ej., seres vivos, suelo, agua, aire). Cada vez es más reconocido que buena parte de los conflictos, que afectan severamente a la humanidad en el presente y la afectarán en el futuro, solo pueden entenderse y gestionarse desde esta perspectiva. Los sistemas socio-naturales que suponen las políticas públicas en medio ambiente y desarrollo sustentable son entidades física y epistemológicamente complejas. Actualmente, el concepto de sistema socio-natural se encuentra bien establecido dentro del dominio de las ciencias naturales (Folke, 2006; Sayles et al., 2019; Ramos-Jiliberto, 2020). Sin embargo, en las ciencias sociales este ha tenido una menor penetración, con notables excepciones (Parson, 1988; Luhmann, 2007). Asimismo, la aplicación del concepto de sistema socio-natural al análisis de política pública es aún incipiente (Jiliberto, 2018; Urquiza, 2015).

El objetivo de este estudio es presentar un conjunto de métodos de modelización y análisis sistémicos orientados al estudio de fenómenos socio-naturales en contextos de toma de decisión de política pública. Este trabajo se inscribe dentro de diversos esfuerzos por desarrollar una propuesta teórica y metodológica para la generación de modelos útiles para la toma de decisión de política pública en sistemas socio-naturales, situación muy propia de las políticas públicas ambientales y de sustentabilidad (Faucheux et al., 1996; Gallopin, 2003; Jiliberto, 2004; Giampietro y Mayumi, 1997; Meadows et al., 1972; Todorov y Marinova, 2011).

Describir sistemas socio-naturales de interés para la política pública no resulta inmediato por dos hechos relacionados: Por un lado, las políticas públicas en medio ambiente y sustentabilidad deben dar cuenta de situaciones conflictivas en las que participan componentes de diversa naturaleza, por lo pronto económicos, sociales y naturales. Por otro lado, la problemática que suscita la política pública deriva, precisamente, del relacionarse de esos componentes. Como resultado de esto se tiene que la política pública interviene en una "realidad" que por su sistemicidad carece de una descripción analítica propia, como la tienen por separado cada uno de los componentes que en ella participan, sean estos económicos, sociales o naturales. Confirma esta aseveración el hecho de que, a pesar de haber pasado más de tres décadas desde la aparición del concepto de desarrollo sustentable en el así conocido Informe Brundlant (CMMAD, 1987), que de alguna forma apela a esa integración sistémica, aún no se dispone de

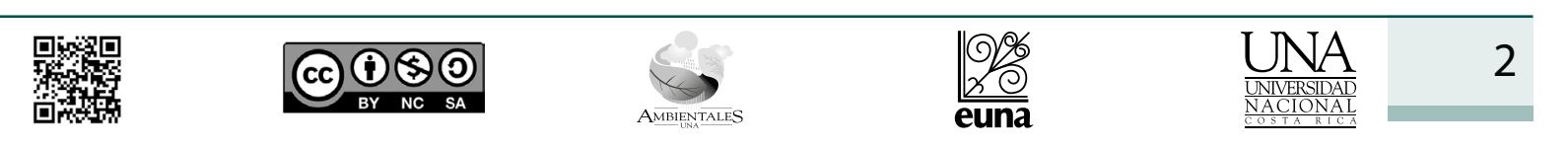




\section{Revista de CIENCIAS AMBIENTALES Tropical Journal of Environmental Sciences}

Revista de Ciencias Ambientales (Trop J Environ Sci) e-ISSN: 2215-3896 (Enero-Junio, 2021) . Vol 55(1): 1-31 DOI: https://doi.org/10.15359/rca.55-1.1

Open Access: www.revistas.una.ac.cr/ambientales e-mail: revista.ambientales@una.ac.cr Ramos-Jiliberto R., Jiliberto-Herrera R.

una definición que permita formular una descripción analítica de la sustentabilidad de alguna entidad "real". (Atkinson et al., 2014, p. 1).

En política pública, como en cualquier contexto de decisión estratégica, no resulta racional tomar decisiones respecto de una entidad $E$ si no se dispone de una descripción razonable del "estado de cosas" en $E$. Por tanto, la ausencia de modelos descriptivos formales del sistema focal para el problema de política pública impone una tarea académica de primera importancia. Este articulo recoge un esfuerzo por disminuir esa brecha mediante la modelización sistémica de los sistemas socio-naturales que suponen las políticas públicas en medio ambiente y desarrollo sustentable. Esta iniciativa se caracteriza por el intento de formular una modelización matemática del sistema socio-natural en cuestión, desde una perspectiva teórica y epistemológica propia. A saber, que aquello modelizado no es una realidad primaria, o positiva, sino la estructura del problema de política pública ambiental o de sustentabilidad, expresado inicialmente como un sistema conceptual o discursivo, el que es luego formalizado matemáticamente.

En este estudio presentamos las siguientes aportaciones: a) un método para mudar un modelo sistémico discursivo/conceptual, apropiado en procesos de formulación de la política pública, en uno formal que facilite la evaluación de opciones de intervención en el sistema socio-natural objeto de la política pública, b) una propuesta de modelización sistémica formal (matemática) de sistemas socio-naturales en contextos decisionales estratégicos, c) elementos de análisis de los sistemas modelizados orientados a la evaluación de escenarios de intervención en dichos sistemas. Las herramientas matemáticas presentadas se enmarcan en los métodos de análisis estructural de sistemas dinámicos y en particular de matrices aleatorias. Esta aproximación metodológica al estudio de los sistemas dinámicos es ampliamente utilizada en el ámbito de las ciencias naturales (Allesina y Tang, 2012; Dambacher y Ramos-Jiliberto, 2007; May, 1972). Finalmente, para ilustrar la utilidad de estas herramientas, se toma como caso de estudio el sistema socio-natural que denominamos sistema de sustentabilidad energía-territorio, de la Región de Coquimbo, Chile, y se evalúa comparativamente el desempeño esperado de medidas de gestión tendientes a producir mejoras estructurales en el sistema de interés.

\section{Metodología}

\subsection{El objeto de estudio: Sistema de sustentabilidad energía-territorio de la Región de Coquimbo, Chile}

La región de Coquimbo, en Chile, está ubicada entre los $29^{\circ}$ y los $32^{\circ}$ de latitud sur, y tiene una superficie aproximada de $40579 \mathrm{~km}^{2}$. El año 2016 disponía de una población de 782801 habitantes. La minería constituye una de las actividades económicas claves de la región, concentrando un $24 \%$ del producto interno bruto (PIB) regional, a mucha distancia de la siguiente actividad económica, servicios personales (turismo) que concentran un $13 \%$ del PIB regional. La actividad de generación y transmisión de energía y agua genera solamente un $3 \%$ del PIB

\begin{tabular}{|c|c|}
\hline 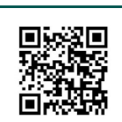 & (c) (i) () (2) \\
\hline
\end{tabular}




\section{Revista de CIENCIAS AMBIENTALES Tropical Journal of Environmental Sciences}

Revista de Ciencias Ambientales (Trop J Environ Sci) e-ISSN: 2215-3896 (Enero-Junio, 2021) . Vol 55(1): 1-31 DOI: https://doi.org/10.15359/rca.55-1.1

Open Access: www.revistas.una.ac.cr/ambientales e-mail: revista.ambientales@una.ac.cr Ramos-Jiliberto R., Jiliberto-Herrera R.

regional, a pesar de la alta demanda de energía eléctrica que produce la gran minería que caracteriza a la región, la que absorbe el $66 \%$ de todo el consumo eléctrico regional.

A pesar de que la generación eléctrica, en la región ha aumentado, exponencialmente, en los últimos años, en particular la renovable no convencional y en especial la eólica solamente un $17 \%$ de la demanda es satisfecha con recursos regionales propios. La región cuenta con importantes potenciales de energía solar y eólica por desarrollar. Según estimaciones oficiales, un $10 \%$ del territorio regional, unos $5800 \mathrm{~km}^{2}$, tiene potencial para desarrollar algún tipo de producción energética solar económicamente viable. No todo ese potencial es factible transformarlo en proyectos, pues obviamente el territorio tiene otros usos y vocaciones que impiden convertir todo ese potencial en energía. No obstante, si se considera solo el potencial renovable en aquellos territorios donde no hay ninguna restricción ni productiva, ni social, ni ambiental para su desarrollo, su realización multiplicaría por nueve la capacidad instalada regional de generación eléctrica.

Debido a las excelentes condiciones de viento y radiación que se presentan en el territorio, se espera que la generación con base en energías renovables no convencionales se incremente a corto plazo, incluso pudiendo por sí misma duplicar la capacidad instalada regional actual al año 2030. Ello haría que la región pudiese transformarse en una región exportadora de energía. Sin embargo, ello no es un proceso automático, pues hay que superar barreras y dificultades que han impedido que hasta ahora se haya producido un crecimiento más acelerado del sector. Por esta razón, el Ministerio de Energía ha elaborado el Plan Energético Regional (PER) de la Región de Coquimbo en Chile como un "... instrumento que busca orientar al sector, en coherencia con las características, vocaciones y aptitudes que ofrezcan los territorios..." (Ministerio de Energía, 2018, p. 18), y que concluye con propuestas sustentables de ordenamiento territorial energético que ayudan a superar los retos sectoriales y favorecen un desarrollo sostenido de las energías renovables no convencionales.

Para asegurar la sustentabilidad de las propuestas de ordenamiento territorial energético se han considerado tempranamente durante el proceso de diseño de las propuestas de política pública variables sociales, ambientales y territoriales, así como de un amplio proceso participativo. Para esos efectos se modelizó la sustentabilidad de la implantación del desarrollo territorial en la región desde una perspectiva sistémica, que intenta capturar el patrón estructural de relacionamiento que históricamente han construido entre ellos el componente energético, el territorial, el ambiental y el social. Se parte del entendido de que justamente la sustentabilidad de una propuesta de ordenamiento territorial del desarrollo energético en la región se deriva de la superación de los aspectos negativos que presenta hoy ese patrón estructural. Como apoyo a ese propósito analítico se elaboró, durante la fase de diagnóstico de la elaboración del PER, lo que se denominó el sistema de sustentabilidad energía-territorio (SSET) de la región de Coquimbo, Chile, cuya funcionalidad era facilitar una mirada de sustentabilidad estructural a las dificultades de implantación territorial del sistema energético en la región, con el propósito de ayudar a la formulación de opciones de desarrollo territorial energético sustentables (Deuman S.A., y Tau Consultora Ambiental S.L. 2017). El SSET de la región de Coquimbo es el sistema socio-natural

\begin{tabular}{|c|c|c|c|c|c|}
\hline 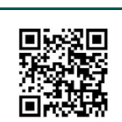 & (c) (1) () (2) & $\Leftrightarrow$ & 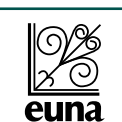 & 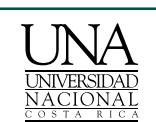 & 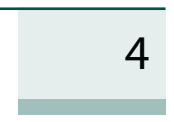 \\
\hline
\end{tabular}




\section{Revista de CIENCIAS AMBIENTALES Tropical Journal of Environmental Sciences}

Revista de Ciencias Ambientales (Trop J Environ Sci) e-ISSN: 2215-3896 (Enero-Junio, 2021) . Vol 55(1): 1-31 DOI: https://doi.org/10.15359/rca.55-1.1

Open Access: www.revistas.una.ac.cr/ambientales e-mail: revista.ambientales@una.ac.cr Ramos-Jiliberto R., Jiliberto-Herrera R.

que se ha escogido para ilustrar la metodología de modelización y análisis desarrollados. Para estos efectos se ha utilizado una versión simplificada del SSET original (23 de 31 elementos).

Entre la modelización inicial del SSET de la región de Coquimbo, cuyo propósito es fundamentalmente discursivo, y la utilizada para la modelización matemática desarrollada en este artículo, media un proceso de formalización de las variables para adaptarlas a las exigencias de la modelización matemática, pero conservando la coherencia discursiva inicial del modelo. El desarrollo de la modelización sistémica del SSET de la región de Coquimbo descansó en los siguientes pasos: a) un proceso de identificación preliminar de temas claves (TC), con base en fuentes secundarias (estudios, análisis políticos, diagnósticos, tesis existentes) y entrevistas con actores claves e informadores cualificados, b) un proceso de validación de TC con los actores relevantes, en general mediante procesos participativos amplios, transparentes e informados, c) un proceso de respaldo de TC mediante una compilación de la evidencia e información que respalda la formulación del TC, d) modelización del sistema del problema de política pública, consistente en establecer las relaciones de dependencia entre TC, los que al interior del modelo se trasforman en elementos y e) proceso de validación de la modelización del sistema, en general mediante procesos participativos amplios, transparentes e informados.

\subsection{Supuestos de la modelización}

La modelización formal del SSET de la región de Coquimbo, adoptada en este estudio, útil a la toma de decisión estratégica, descansa en un conjunto de supuestos. En primer lugar, se trata de la modelización de una estructura causal. Esto es, de un conjunto de elementos que se entiende han construido entre si una organización funcional estable. Es decir, asumimos que los elementos del sistema mantienen valores acotados en el tiempo, y la intensidad de sus relaciones de influencia ha alcanzado una magnitud aproximadamente constante. Los elementos del sistema modelizado corresponden a variables cuantitativas continuas, medidas en escala de intervalo. Esto es, las variables pueden asumir cualquier valor, positivo o negativo, y el valor cero en un valor posible más, sin indicar ausencia de la característica. Consistente con la perspectiva sistémica, cada elemento de este sistema solo tiene sentido a la luz de sus relaciones con los otros elementos. Estas relaciones, expresadas en el sistema modelizado, corresponden a relaciones de influencia directa, donde los cambios que eventualmente puedan transmitirse desde una variable (el emisor de la influencia) hacia otra (el receptor), se asume ocurren sin intervención de terceros (ver Vázquez et al., 2015). En la sección siguiente se presentan, de manera formal, los elementos, estructuras y procedimientos utilizados en nuestra modelización y análisis de sistemas socio-naturales, que aplicaremos al SSET de la región de Coquimbo.

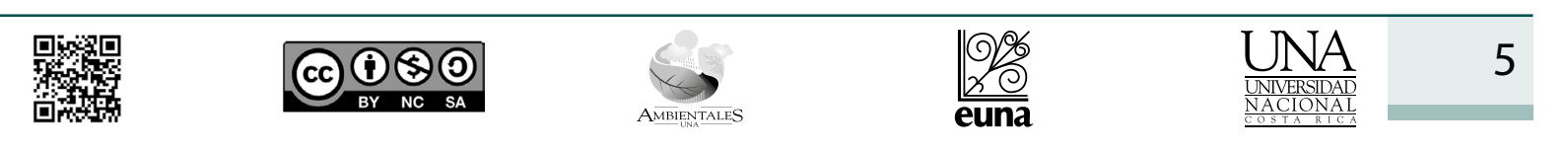




\section{Revista de CIENCIAS AMBIENTALES Tropical Journal of Environmental Sciences}

Revista de Ciencias Ambientales (Trop J Environ Sci) e-ISSN: 2215-3896 (Enero-Junio, 2021) . Vol 55(1): 1-31 DOI: https://doi.org/10.15359/rca.55-1.1

Open Access: www.revistas.una.ac.cr/ambientales e-mail: revista.ambientales@una.ac.cr Ramos-Jiliberto R., Jiliberto-Herrera R.

\subsection{Modelización del sistema: El digrafo y la matriz de influencias}

Las relaciones de influencia directas entre los elementos del sistema modelizado pueden representarse gráficamente en un digrafo ponderado (ver Apéndice 1, Figura A1.1). En general, un grafo es una representación formal de una red de elementos (los vértices o nodos del sistema) y sus relaciones de influencia directa (las aristas o vínculos entre vértices). Cuando las aristas indican la dirección de la influencia, $A \rightarrow B$ indica que $A$ influye a $B$ pero no viceversa, el grafo se denomina "grafo direccionado" o más simplemente "digrafo". Cuando las aristas indican la magnitud o peso de la influencia, el grafo se denomina "grafo ponderado", también llamado "grafo pesado" o "grafo etiquetado". Así, un digrafo ponderado corresponde a un grafo en que se indica la dirección y el peso de las relaciones de influencia entre los vértices. Para el caso particular de los sistemas socio-económicos que nos ocupan en este estudio, los elementos de un sistema y, consecuentemente, los vértices del grafo que lo representan serán variables dinámicas, indicadas por el conjunto $X=\left\{x_{1}, x_{2}, x_{3}, \ldots, x_{n}\right\}$, a las que se les asocia una valoración ética $V=\left\{v_{1}, v_{2}, v_{3}, \ldots, v_{n}\right\}$ positiva $\left(v_{i}=1\right)$ o negativa $\left(v_{i}=-1\right)$ que indica si un aumento en el valor de dicha variable es o no deseable, basado en la provisión de bienes o servicios que se derivan de ella. Por otro lado, cada arista $m_{i j}$ conecta el par de vértices $x_{i}$ y $x_{j}$ y se definen como:

$$
m_{i j}=\left.\frac{\partial\left(\frac{d x_{i}}{d t}\right)}{\partial x_{j}}\right|_{x^{*}} \text { (E. 1) }
$$

esto es, el cambio en la tasa de incremento de $x_{i}$ tras un aumento en el valor de $x_{j}$, evaluado en torno al equilibrio $x^{*}$ del sistema y manteniendo fijas el resto de las variables del sistema.

Una representación equivalente del digrafo ponderado es la otorgada por la matriz de influencias (Apéndice 1, Figura A1.1). La matriz de influencias es análoga a la matriz comunitaria, que posee gran tradición en ecología teórica (Novak et al., 2016) y particularmente en el análisis de sistemas ecológicos complejos (Puccia y Levins, 1985; Dambacher y Ramos-Jiliberto, 2007). La matriz de influencias $M$ tiene tamaño $n \times n$, siendo $n$ el número de variables del sistema. Sus elementos $m_{i j}$ se definen como en la Ecuación 1, es decir, de igual manera que las aristas del digrafo ponderado equivalente.

\subsection{Análisis prospectivo del sistema: Matriz de efectos netos}

Una característica general de los sistemas complejos de cualquier índole es la transmisión de cambios entre variables que no están necesariamente conectadas en forma directa. En el ejemplo de la Figura A1.1, la variable $x_{2}$ influye directamente a $x_{3}$ con intensidad 5, pero también influye indirectamente a $x_{3}$ vía influir negativamente a $x_{5}$ con intensidad -4 encadenado con la influencia negativa de $x_{5}$ a $x_{3}$ con magnitud -5 . De hecho, es fácil percatarse gráficamente en el sistema de Figura A1.1 que cada variable influye, directa o indirectamente, en todas las otras

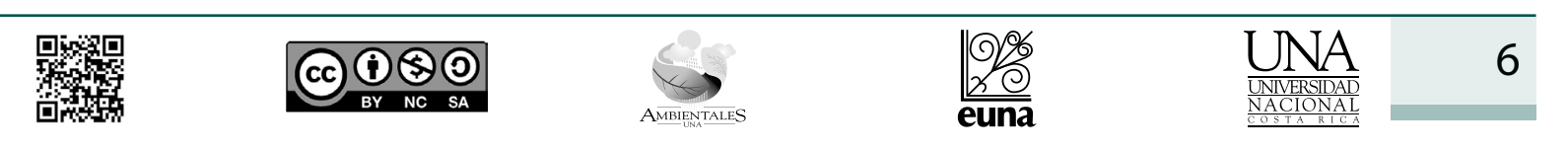




\section{Revista de CIENCIAS AMBIENTALES Tropical Journal of Environmental Sciences}

Revista de Ciencias Ambientales (Trop J Environ Sci) e-ISSN: 2215-3896 (Enero-Junio, 2021) . Vol 55(1): 1-31 DOI: https://doi.org/10.15359/rca.55-1.1

Open Access: www.revistas.una.ac.cr/ambientales e-mail: revista.ambientales@una.ac.cr Ramos-Jiliberto R., Jiliberto-Herrera R.

variables del sistema. También es posible darse cuenta de que el análisis visual de las cadenas de influencias es insuficiente para sistemas de muchas variables.

A fin de entender la acción conjunta de estas relaciones complejas, es posible obtener todas las influencias netas, las que integran todas las influencias directas e indirectas ejercidas por una variable sobre otra, entre todas las variables del sistema. Esto se realiza según la matriz de efectos netos $A$ de tamaño $n \times n$, cuyos elementos $a_{i j}$ representan

$$
a_{i j}=\frac{\partial x_{i}^{*}}{\partial P_{j}} \text { (E. 2) }
$$

en que $x_{i}^{*}$ es el valor de equilibrio de la variable $x_{i}$ y $P_{j}$ es una presión sostenida ejercida sobre la variable $x_{j}$. Por lo tanto, el elemento $a_{i j}$ representa el cambio esperado a largo plazo en el valor de equilibrio de una variable $x_{i}$ producto de una presión permanente y constante ejercida sobre la variable $x_{j}$. Los efectos netos especificados en $A$ se generan como resultado del conjunto de relaciones directas e indirectas entre las variables del sistema. El cálculo de $A$ se realiza a partir de la matriz comunitaria de la forma:

$$
A=-M^{-1} \text { (E. 3) }
$$

Proponemos 4 nuevas métricas que consideramos útiles para el análisis de los sistemas socio-naturales. Estas métricas, las cuales se detallan en el Apéndice 3, son: sensibilidad $S$, efectividad $K$, virtuosismo $U$ y reactividad positiva $R$. Estas métricas están basadas en la matriz de efectos netos $A$, e indican el potencial de cada variable del sistema en cuanto receptora $(S$ y $R$ ) y transmisora $(K$ y $U)$ de cambios. Esta perspectiva contrasta con las más conocidas medidas de centralidad, las cuales caracterizan la importancia de las variables de acuerdo con su posición dentro de la red y se calculan a partir de las relaciones (i.e. influencias) directas entre los elementos del sistema.

\subsection{Simulación computacional}

Para analizar el sistema socio-natural en estudio se construyeron códigos computacionales en lenguaje MATLAB (The MathWorks Inc., Natick MA). Los pasos del procedimiento utilizado fueron: 1) Asignar valores aleatorios a los elementos $m_{i j}$ de la matriz de influencias $M$. Previo a todo análisis, la matriz se re-escala de modo que los valores de sus elementos asuman valores entre 0 y 1 . Luego, cada matriz aleatoria se obtiene multiplicando sus elementos ubicados fuera de la diagonal, que indican efectos entre variables diferentes, por un valor aleatorio muestreado desde una distribución uniforme dentro del intervalo $(1 / 2,2)$. A los elementos ubicados dentro de la diagonal, que representan auto-amortiguamiento, se les asigna un valor arbitrario $a_{i i}<0$, suficientemente pequeño para obtener una matriz estable (ver paso siguiente). Tal valor dependerá de los valores absolutos de las celdas de la matriz y, por tanto, de la

\begin{tabular}{|c|c|}
\hline 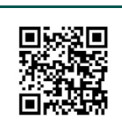 & (c) (i) () (2) \\
\hline
\end{tabular}
escala en la cual se expresen las magnitudes de las influencias (ver Haydon 1994). En nuestro 


\section{Revista de CIENCIAS AMBIENTALES Tropical Journal of Environmental Sciences}

Revista de Ciencias Ambientales (Trop J Environ Sci) e-ISSN: 2215-3896 (Enero-Junio, 2021) . Vol 55(1): 1-31 DOI: https://doi.org/10.15359/rca.55-1.1

Open Access: www.revistas.una.ac.cr/ambientales e-mail: revista.ambientales@una.ac.cr Ramos-Jiliberto R., Jiliberto-Herrera R.

caso, fijamos todos los elementos $a_{i i}=-3$. Cada matriz aleatoria construida a partir de $M$ la designaremos como $\widehat{M}$.2) Ejecutar prueba de estabilidad local. Un sistema continuo especificado en la matriz $\widehat{M}$ de tamaño $n \times n$ se considera estable localmente cuando, tras ser perturbada ligeramente desde una situación de equilibrio, el sistema tiende a retornar a dicho equilibrio. Esto se verifica matemáticamente cuando todos y cada uno de los autovalores de la matriz $\widehat{M}$ (en caso de que estos sean números reales) o su parte real (en caso de que sean números complejos) son estrictamente negativos (p.ej., May, 1972). Los autovalores se obtienen resolviendo la ecuación característica $\operatorname{det}(\widehat{M}-\lambda I)=0$, donde $I$ corresponde a la matriz de identidad. Este criterio es estándar en teoría de sistemas dinámicos y no es válido cuando la perturbación es de gran magnitud. Alternativamente a las estimaciones numéricas, para verificar la negatividad de los autovalores puede utilizarse el criterio de Routh-Hurwitz, basado en los coeficientes del polinomio característico. Este criterio es particularmente útil cuando la matriz de influencias y se expresa de forma simbólica (Dambacher et al., 2003). 3) Si $\widehat{M}$ es estable, se calcula su correspondiente matriz de efectos netos $A$. 4) Este proceso se repite $r$ veces (aquí usamos $r=5000$ ). De este modo obtenemos $r$ matrices $A$, calculadas a partir de $r$ matrices $\widehat{M}$, todas con elementos aleatorios. 5) Se obtiene una matriz de efectos netos promedio $\bar{A}$, cuyas celdas $\bar{a}_{i j}$ son el promedio de las $r$ celdas $a_{i j}$. 6) Se reescalan los valores de $\bar{A}$, y se obtiene la matriz $\hat{A}$, con elementos $\hat{a}_{i j} \in[0,1]$. 7) Se categorizan los valores $\hat{a}_{i j}$ para obtener la matriz $\dot{A}$, con elementos:

$$
\dot{a}_{i j}\left\{\begin{array}{ccc}
-2, & \text { si } & \hat{a}_{i j} \leq-U_{H} \\
-1, & \text { si } & -U_{H}<\hat{a}_{i j} \leq-U_{L} \\
0, & \text { si } & -U_{L}<\hat{a}_{i j}<U_{L} \\
1, & \text { si } & U_{L} \leq \hat{a}_{i j}<U_{H} \\
2, & \text { si } & \hat{a}_{i j} \geq U_{H}
\end{array}\right.
$$

con valores umbrales $U_{L}=0.01$ y $U_{H}=0.7$. Así, la matriz $\dot{A}$ muestra los efectos netos esperados para el sistema especificado en $M$, categorizados en nulos $\left(\dot{a}_{i j}=0\right)$, reforzantes leves $\left(\dot{a}_{i j}=1\right)$, reforzantes fuertes $\left(\dot{a}_{i j}=2\right)$, inhibitorios leves $\left(\dot{a}_{i j}=-1\right)$ e inhibitorios fuertes $\left(\dot{a}_{i j}=-2\right)$. A partir de esta matriz de resultados, se calculan las métricas propuestas previamente $(S, K, R, U)$.

\subsection{Análisis de intervención}

Es posible proyectar las consecuencias de medidas de intervención ejercidas sobre un conjunto de variables del sistema. Este análisis es útil, por un lado, para evaluar medidas en curso implementadas como política pública, o para evaluar acciones espontáneas o no planificadas ejercidas por la ciudadanía u organizaciones civiles, o bien para evaluar una posible estrategia de gestión. A modo de ejemplo (Apéndice 1, Figura A1.2), supóngase que se quiere evaluar una intervención consistente en una estrategia de gestión compuesta por tres presiones simultáneas: una presión inhibitoria fuerte (magnitud -10) sobre la variable más una presión inhibitoria débil

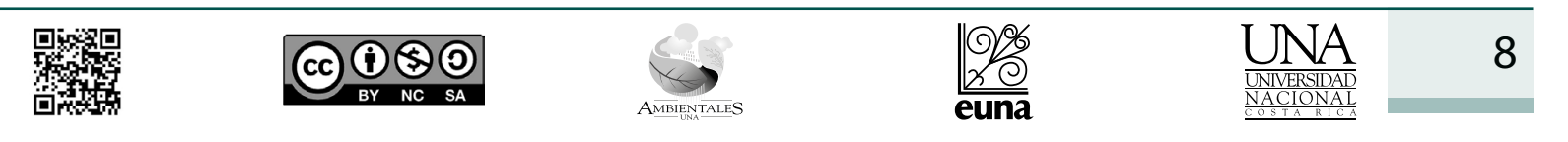




\section{Revista de CIENCIAS AMBIENTALES Tropical Journal of Environmental Sciences}

Revista de Ciencias Ambientales (Trop J Environ Sci) e-ISSN: 2215-3896 (Enero-Junio, 2021) . Vol 55(1): 1-31 DOI: https://doi.org/10.15359/rca.55-1.1

Open Access: www.revistas.una.ac.cr/ambientales e-mail: revista.ambientales@una.ac.cr Ramos-Jiliberto R., Jiliberto-Herrera R.

(magnitud -2) sobre la variable más una presión reforzadora moderada (magnitud 5) sobre la variable. Esto se traduce en modificar la matriz de influencias $M$ para obtener $M^{I}$ (Apéndice 1, Figura A1.2). Nótese que la estrategia de intervención se representa mediante la adición de una nueva variable al sistema, con sus correspondientes presiones sobre las variables preexistentes. Además, puede asumirse que existe reforzamiento o inhibición, ejercidas por algunas variables de sistema, sobre la aplicación de la intervención. Una vez definida $M^{I}$, procedemos a evaluar los efectos de la intervención a través del análisis de la matriz de efectos netos, calculada mediante Ec. 3 usando $M^{I}$. Llamaremos efectos primarios al efecto neto (de valor -2, -.1, 0, 1 o 2) esperado a largo plazo en las variables del sistema, generado por la aplicación de una intervención. Seguido, se calcularán las métricas $S, R, K$ y $U$ sobre las matrices $M$ y $M^{I}$ (luego de extraída la fila y columna que representa la intervención), a fin de evaluar las diferencias generadas por la aplicación de la intervención. En este estudio, las intervenciones se aplicaron con intensidad igual a -3 para todas las variables afectadas. Cambios dentro del orden de magnitud no afectaron cualitativamente nuestros resultados.

\section{Resultados}

\subsection{Identificación de elementos del sistema modelizado}

En la Figura 1 se presentan los 20 temas claves (TC) identificados, que constituyen los elementos de nuestro modelo sistémico. En el Apéndice 2 se provee una descripción de dichos TC.

\subsection{El modelo del SSTE de Coquimbo}

El SSET es representado por el digrafo con signos presentado en Figura 1. El sistema representado incluye 20 variables de sistema y 40 relaciones de influencia. De estas, 11 son influencias negativas y 29 son positivas. La conectancia (porcentaje de interacciones posibles efectivamente realizadas en el sistema) es de $11 \%$. El sistema es totalmente conexo en sentido débil. Es decir, existe al menos un camino que conecta todas las variables entre sí. Además, todas las variables, excepto la 19 (la cual no presenta influencias de entrada), conforman una unidad conexa en sentido fuerte, es decir, existen caminos en ambos sentidos que conectan las variables de dicha unidad.

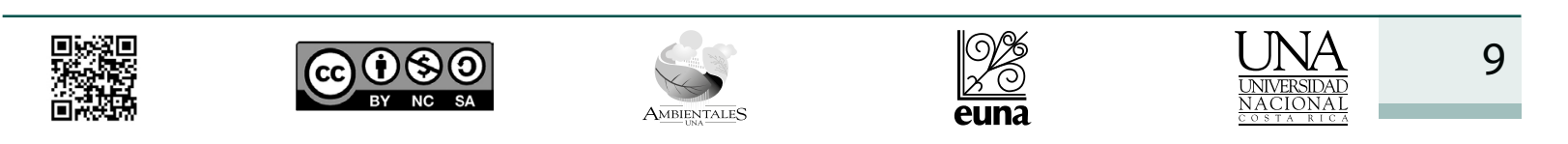




\section{Revista de CIENCIAS AMBIENTALES Tropical Journal of Environmental Sciences}

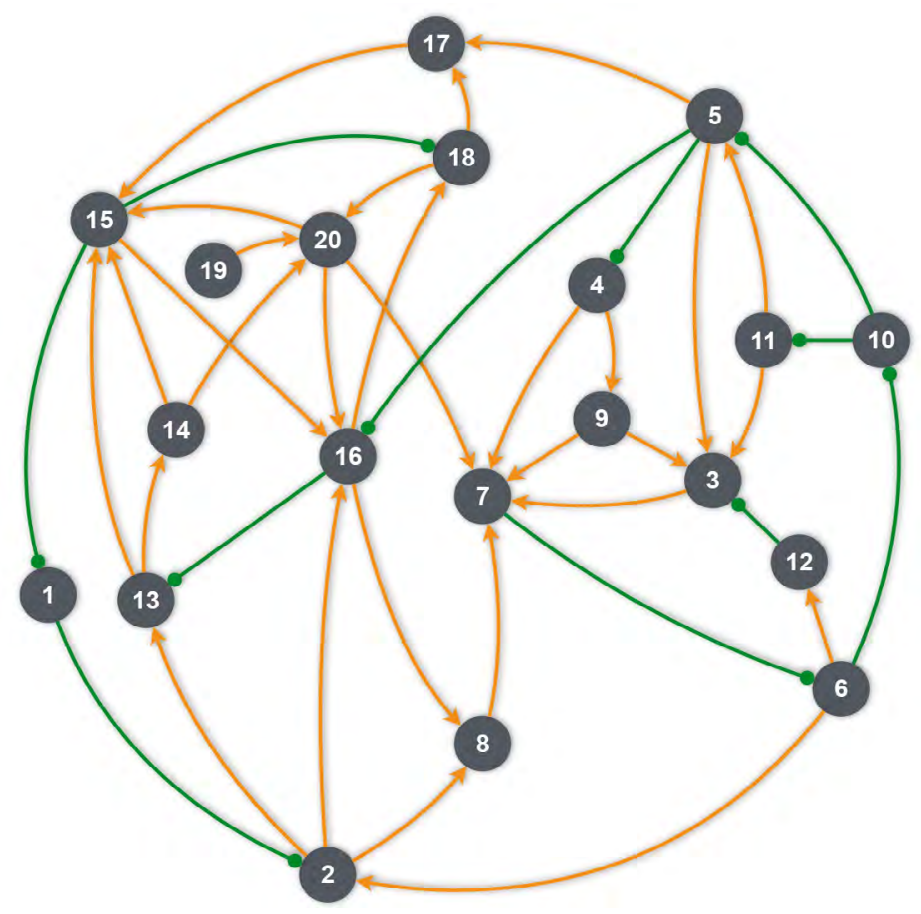

Figura 1. Digrafo que representa nuestro modelo del sistema SSET. Los arcos terminados en punta de flecha (en naranja) señalan influencias positivas. Los arcos terminados en círculo (en verde) señalan influencias negativas. Las siguientes son las variables de sistema correspondientes a cada rótulo usado en el digrafo. 1: Nivel de energización rural, 2: déficit energético y pobreza rural, 3: nivel de desconfianza de la comunidad frente a proyectos de energía, 4: condicionamiento territorial para el desarrollo energético, 5: escasez y conflictividad del recurso hídrico, 6: debilidad institucional, 7: dificultades para el desarrollo de las energías renovables, 8: descapitalización del mundo rural, 9: riesgos ambientales de las energías renovables, 10: nivel de gestión de cuencas hidrográficas, 11: sequía y desertificación, 12: nivel de insatisfacción frente a procesos participativos, 13: despoblamiento rural, 14: concentración de la población en la enconurbación Serena-Coquimbo, 15: nivel de desintegración funcional del territorio, 16: monoproductividad de economías territoriales, 17: desarticulación económica y territorial minera, 18: autonomía territorial minera, 19: concentración territorial de la infraestructura energética, 20: concentración territorial de la demanda.

Figure 1. Digraph representing our SSET system model. Arrow-ending arcs (orange) indicate positive influences. Circle-ended arcs indicate negative influences. The following system variables correspond to each label used in the digraph. 1: Level of rural energization, 2: energy shortages and rural poverty, 3: level of distrust of the community against energy projects, 4: territorial conditioning for energy development, 5: scarcity and contentiousness of the water resource, 6: institutional weakness, 7: difficulties for the development of renewable energies, 8: decapitalization of rural areas, 9: environmental risks of renewable energy, 10: level of watershed management, 11: drought and desertification, 12: level of dissatisfaction with participatory processes, 13: rural depopulation, 14: concentration of the population in Serena-Coquimbo conurbation, 15: level of functional disintegration of the territory, 16: monoproductivity of territorial economies, 17: economic and territorial disarticulation due to mining, 18: mining territorial autonomy, 19: territorial concentration of energy infrastructure, 20: territorial concentration of demand.

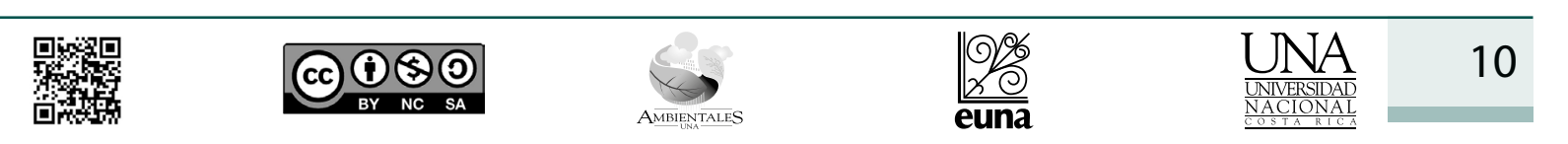




\section{Revista de CIENCIAS AMBIENTALES Tropical Journal of Environmental Sciences}

Revista de Ciencias Ambientales (Trop J Environ Sci) e-ISSN: 2215-3896

(Enero-Junio, 2021) . Vol 55(1): 1-31 DOI: https://doi.org/10.15359/rca.55-1.1

Open Access: www.revistas.una.ac.cr/ambientales e-mail: revista.ambientales@una.ac.cr Ramos-Jiliberto R., Jiliberto-Herrera R.

\subsection{Análisis de intervención sobre el SSET}

Para nuestro sistema de interés, analizaremos las consecuencias proyectadas de los siguientes escenarios hipotéticos de intervención (Cuadro 1).

Cuadro 1. Escenarios alternativos de intervención aplicadas sobre el modelo de SSET de Coquimbo. En ambos casos se asumió que la variable de sistema 6 (debilidad institucional) inhibe la aplicación de la intervención.

Table 1. Alternative intervention scenarios applied on the SSET Coquimbo model. In both cases it was assumed that the system variable 6 (institutional weakness) inhibits the intervention application.

\begin{tabular}{lll}
\hline \multicolumn{1}{c}{ Variable de sistema } & \multicolumn{1}{c}{ Escenario 1 } & \multicolumn{1}{c}{ Escenario 2 } \\
\hline 2. Déficit energético y pobreza rural & Inhibición & - \\
3. Nivel de desconfianza de la comunidad frente a proyectos de energía & - & Inhibición \\
5. Escasez y conflictividad del recurso hídrico & Inhibición & - \\
6. Debilidad institucional & Inhibición & - \\
8. Descapitalización del mundo rural & - & Inhibición \\
9. Riesgo ambiental de ER & - & Inhibición \\
12. Nivel de insatisfacción frente a procesos participativos & - & Inhibición \\
13. Despoblamiento rural & Inhibición & - \\
\hline
\end{tabular}

El escenario 1 apuesta por una intervención con características proactivas tendiente a incidir en algunos de los elementos "duros" del sistema, como son el despoblamiento rural, el déficit energético y la pobreza rural o la debilidad institucional; espera que su modificación contribuya positivamente a los elementos "finalistas" para el PER, como es la propia dificultad para la implantación de las energías renovables en la región. Además, la selección específica de las variables a intervenir se llevó a cabo considerando los indicadores estructurales del sistema que se proponen en este artículo (sensibilidad, efectividad, virtuosismo, reactividad positiva) para analizar un sistema socio natural sujeto de intervención. De esta forma la modelización inicial y su análisis estructural tributan al diseño de las opciones de intervención.

El escenario 2 simula una intervención más blanda, pues afecta elementos más sociales y perceptivos del sistema, como son nivel de desconfianza de la comunidad frente a proyectos, nivel de insatisfacción frente a procesos participativos, riesgo ambiental de ER, y descapitalización del mundo rural (entendido básicamente como escasez de capital humano) y que puede leerse como una apuesta que requiere menos recursos, y quizás una aplicación menos sostenida en el tiempo, salvo por la apuesta a disminuir. Para la selección de variables a intervenir en este escenario, no se consideraron los indicadores cuantitativos estructurales.

En síntesis, el escenario 1 puede leerse como una intervención más proactiva y de largo plazo, informada por indicadores cuantitativos. En tanto, el escenario 2 puede leerse como uno más cortoplacista que apuesta por intervenir en los elementos políticos y sociales del sistema para mejorar su eficiencia, sin orientaciones cuantitativas. Ambos escenarios se han creado exclusivamente para este ejercicio.

\begin{tabular}{|c|c|c|c|c|c|}
\hline 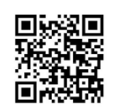 & (c) (1) () (2) & $\underset{\text { AMBENENALIES }}{\infty}$ & $\frac{1 \%}{\text { euna }}$ & 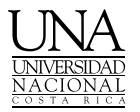 & 11 \\
\hline
\end{tabular}




\section{Revista de CIENCIAS AMBIENTALES Tropical Journal of Environmental Sciences}

Revista de Ciencias Ambientales (Trop J Environ Sci) e-ISSN: 2215-3896 (Enero-Junio, 2021) . Vol 55(1): 1-31 DOI: https://doi.org/10.15359/rca.55-1.1

Open Access: www.revistas.una.ac.cr/ambientales e-mail: revista.ambientales@una.ac.cr Ramos-Jiliberto R., Jiliberto-Herrera R.

\subsection{Resultados del análisis de intervención}

La aplicación del escenario 1, que tuvo como blanco la inhibición de 4 variables de sistema de bajo virtuosismo y alta efectividad, arrojó como proyección un cambio nulo en una variable, 3 cambios débiles valorados negativos y 16 cambios valorados positivos, 4 de los cuales fueron efectos fuertes (Figura 2). Además, se proyectan cambios de variada intensidad y sentido en las propiedades SRKU de las variables del sistema (Figura 3). En promedio, la tendencia de las variables del sistema es a incrementar el valor de las cuatro variables SRKU como producto de la intervención 1, lo cual indicaría que el sistema se torna más propenso a cambios valorados positivos, en un contexto de eventuales perturbaciones futuras. Como resultado de la intervención, todos los elementos propiamente energéticos del sistema mejoran su situación, salvo el elemento "concentración de la infraestructura energética" que se muestra más resistente al cambio.
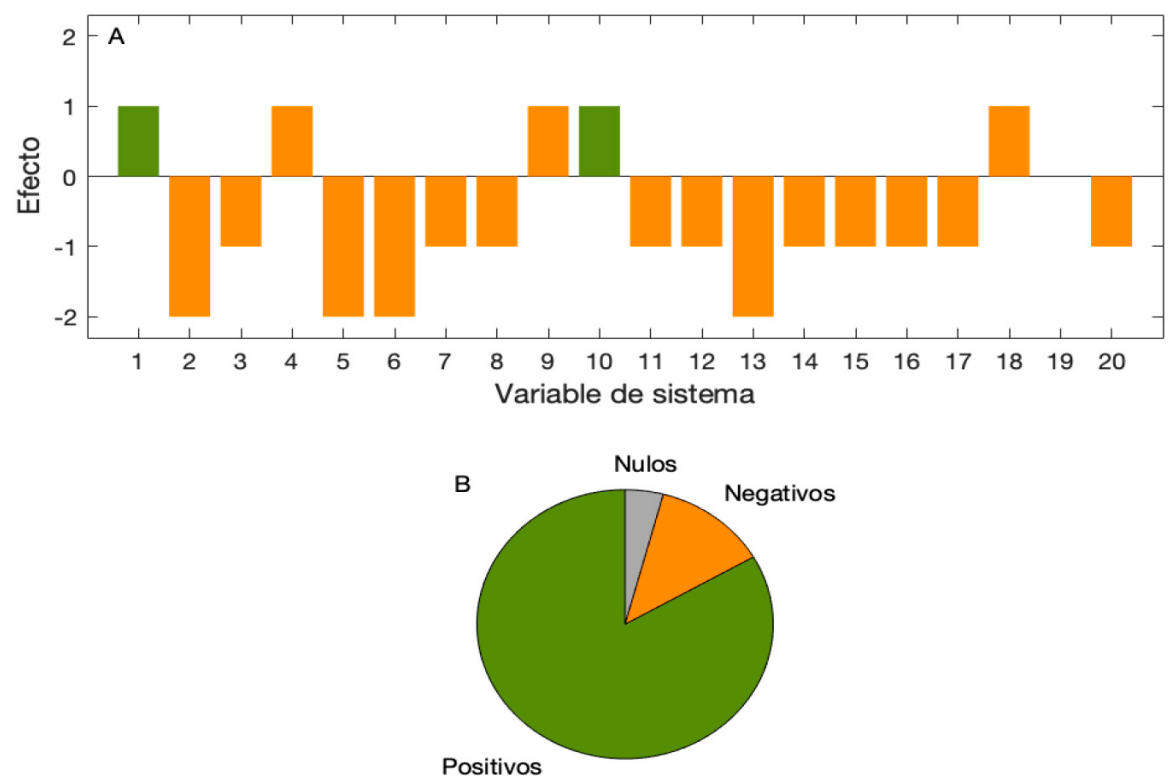

Figura 2. Efectos primarios del escenario de intervención 1 ejercido sobre el sistema de estudio. En A se muestra el nivel de efecto neto total $(0=$ despreciable, 1 y $-1=$ moderado, 2 y -2 = fuerte $)$ de la intervención 1 sobre cada variable de sistema (1-20). Las barras verdes/naranjas muestras las variables con valoración positiva/negativa. Efectos positivos/negativos indican aumento/disminución final del valor de la variable producto de la intervención 1. En B se muestra la composición ponderada (efectos fuertes cuentan el doble que los moderados) relativa de efectos neutros (sección de torta gris), valorados positivo (sección de torta verde) y valorados negativo (sección de torta naranja) de la intervención 1 sobre las variables del sistema.

Figure 2. Primary effects of intervention scenario 1 exerted on the study system. In A it is shown the total net effect level $(0=$ negligible, 1 and $-1=$ moderate, 2 and $-2=$ strong) of the intervention on each system variable (1-20). Green/orange bars show variables valued as positive/negative. Positive/negative effects indicate a final increase/decrease of the variable level as a product of intervention 1. In B it is shown the relative weighted composition (strong effects count twice as much as moderate ones) of neutral effects (grey pie area), positively valued effects (green pie area) and negatively valued effects (orange pie area) of the intervention 1 on system variables.

(cc)




\section{Revista de CIENCIAS AMBIENTALES Tropical Journal of Environmental Sciences}

Revista de Ciencias Ambientales (Trop J Environ Sci) e-ISSN: 2215-3896

(Enero-Junio, 2021) . Vol 55(1): 1-31 DOI: https://doi.org/10.15359/rca.55-1.1

Open Access: www.revistas.una.ac.cr/ambientales e-mail: revista.ambientales@una.ac.cr Ramos-Jiliberto R., Jiliberto-Herrera R.
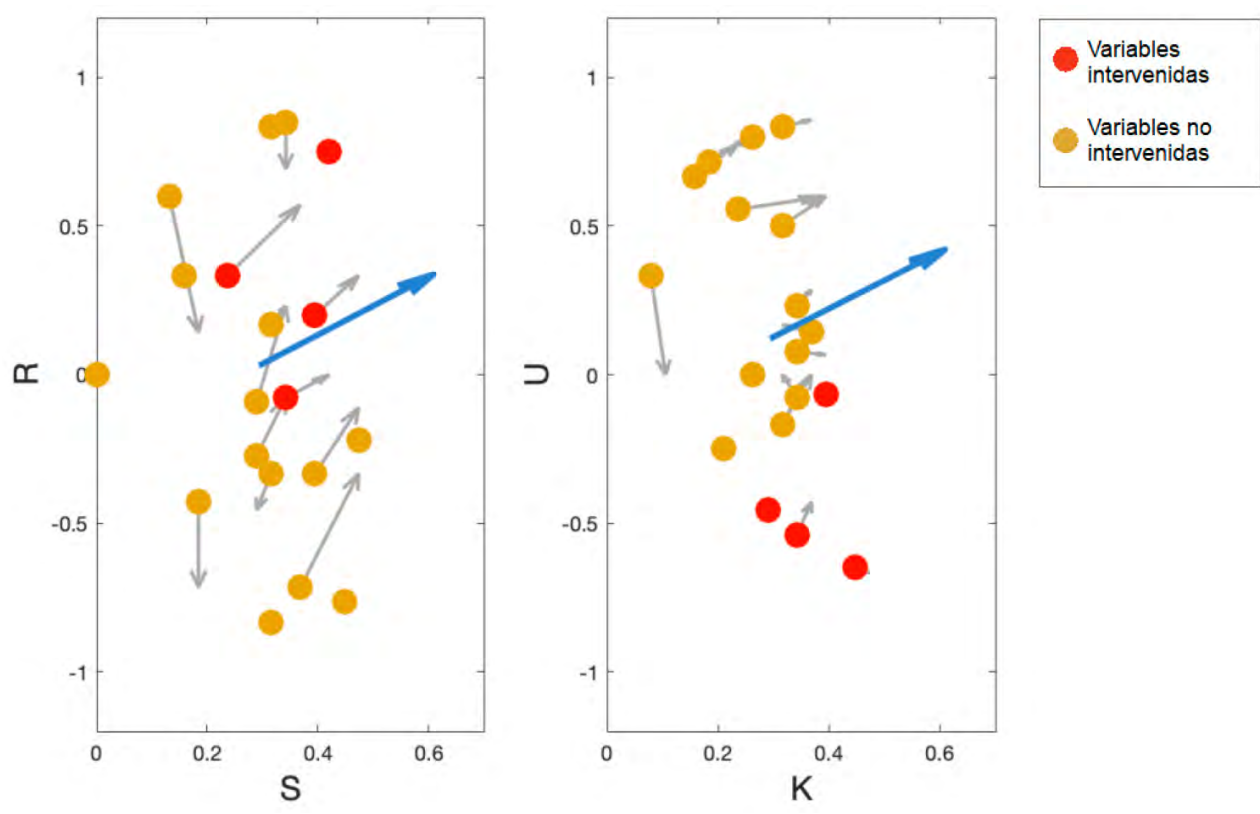

Figura 3. Cambio en propiedades SRKU de las variables de sistema como efecto de la intervención 1 . $\mathrm{S}=$ sensibilidad, $\mathrm{R}$ = reactividad positiva, $\mathrm{K}$ = efectividad, $\mathrm{U}=$ virtuosismo. Los círculos señalan el valor de cada par de propiedades (S, R o K, U) antes de la intervención. Las flechas grises muestran los cambios en los valores de las propiedades producto de la intervención. La flecha grande azul muestra la dirección de cambio en las propiedades promedio del sistema. Su magnitud está reescalada x10 para mejor visualización. Los círculos rojos corresponden a las variables que fueron intervenidas (inhibidas) en el marco de la intervención 1. Los valores para cada variable se presentan en el Apéndice 4.

Figure 3. Shift in SRKU properties of system variables as a product of intervention 1. $S=$ sensitivity, $R=$ positive reactivity, $K=$ effectiveness, $U=$ virtuosity. Circles represent the value of each pair or properties $(S, R$ or $K, U)$ before intervention. Grey arrows show the shifts in average values of system properties, as a product of the intervention. Its magnitude was rescaled tenfold for better visualization. Red circles correspond to perturbed (inhibited) variables as part of intervention 1. The values for each variable are provided in Appendix 4.

La aplicación del escenario 2, que corresponde a una decisión plausible guiada por opinión experta, resulta en una proyección de 8 cambios nulos, 7 cambios débiles valorados negativos y 5 cambios valorados positivos, 2 de los cuales son efectos fuertes (Figura 4). Adicionalmente, las variables del sistema muestran una leve tendencia a aumento de sensibilidad y efectividad y a disminución de reactivadas positiva y virtuosismo producto de la intervención (Figura 5). Esto se traduce en una propensión a cambios negativos en un contexto de eventuales perturbaciones futuras. Como resultado de la intervención todos los elementos propiamente energéticos del sistema, salvo "dificultades para el desarrollo de las ER", no modifican su situación. Es decir, la intervención parece ser poco efectiva en términos de sus objetivos últimos.

\begin{tabular}{|c|c|c|c|c|}
\hline 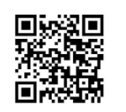 & (c) (1) () (2) & $\underset{\text { AMBENENALIES }}{\infty}$ & $\frac{1 \%}{\text { euna }}$ & 13 \\
\hline
\end{tabular}




\section{Revista de CIENCIAS AMBIENTALES Tropical Journal of Environmental Sciences}
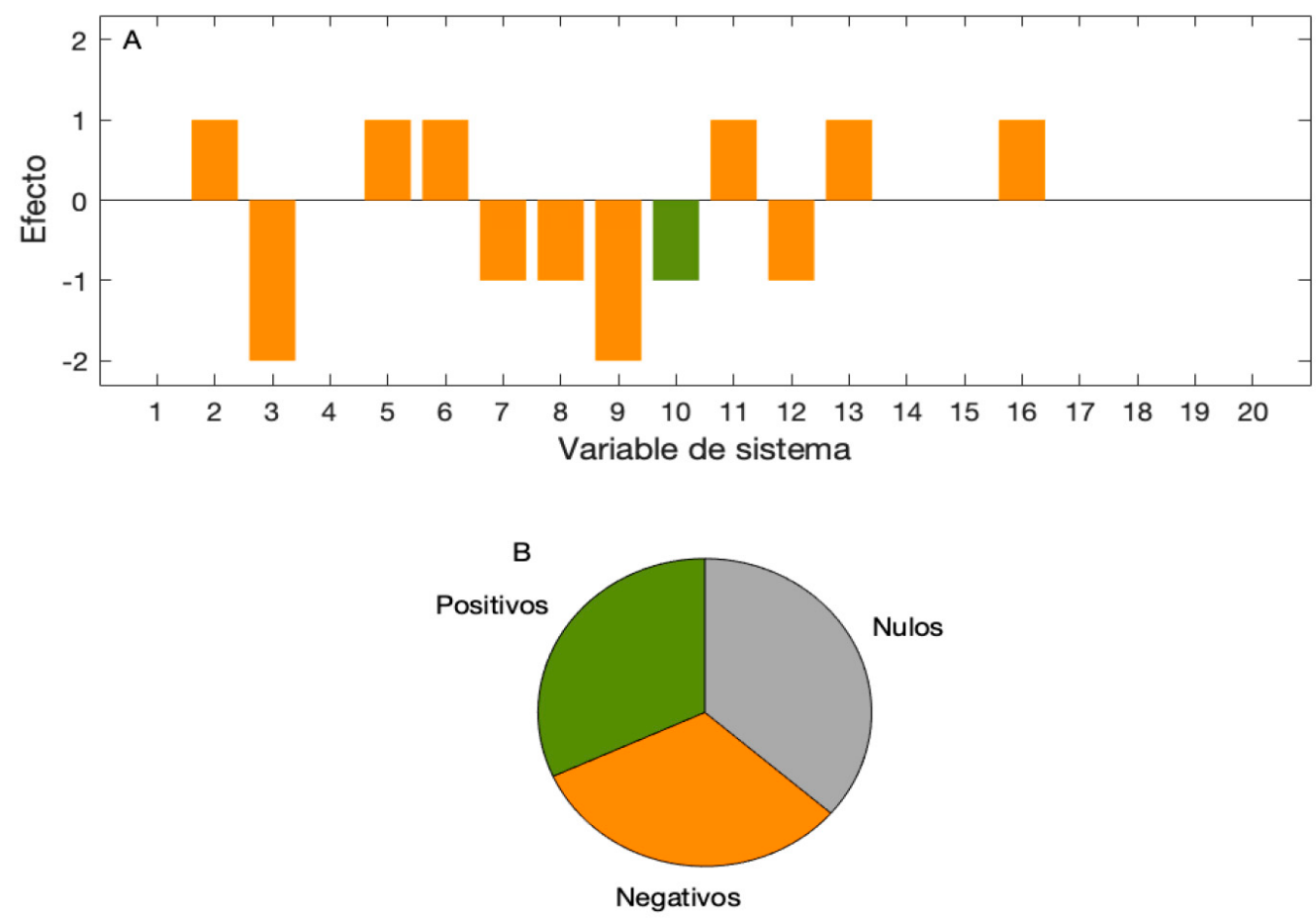

Figura 4. Efectos primarios del escenario de intervención 2 ejercido sobre el sistema de estudio. Ver detalle en Figura 2.

Figure 4. Primary effects of intervention scenario 2 exerted on the study system. See details in Figure 2.

\subsection{Medidas de centralidad}

En el Apéndice 5 se muestran los valores de centralidad direccionada de grado, grado de entrada, grado de salida, intermediación, cercanía de entrada y cercanía de salida, para las variables del sistema. Nótese que estas medidas son conocidas y utilizadas en el contexto de la teoría de redes (Barabási 2016, Newman 2018). Las variables 15 y 16 mostraron el mayor valor de centralidad de grado total, mientras que las variables 7 y 5 presentaron mayor centralidad de grado de entrada y salida, respectivamente. Los mayores valores de centralidad de intermediación los evidenciaron las variables 6 y 7. La variable 7 mostró el mayor valor de centralidad de cercanía de entrada, mientras que las variables 5 y 6 manifestaron los mayores valores para centralidad de cercanía de salida. En general hubo una débil correlación entre los valores $\mathrm{S}, \mathrm{R}$, $\mathrm{K}$ y U presentados aquí y los valores de las medidas de centralidad (Apéndice 6 y 7). Es interesante, sin embargo, que hubo correlaciones fuertes entre la centralidad de cercanía de entrada y la sensibilidad $S(\rho=0.907, \mathrm{P}<0.001)$, así como entre la centralidad de cercanía de salida y la efectividad $K(\rho=0.888, \mathrm{P}<0.001)$.

\begin{tabular}{|c|c|c|}
\hline 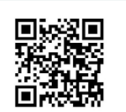 & (c) (1) (9) & 14 \\
\hline
\end{tabular}




\section{Revista de CIENCIAS AMBIENTALES Tropical Journal of Environmental Sciences}

Revista de Ciencias Ambientales (Trop J Environ Sci) e-ISSN: 2215-3896 (Enero-Junio, 2021) . Vol 55(1): 1-31 DOI: https://doi.org/10.15359/rca.55-1.1

Open Access: www.revistas.una.ac.cr/ambientales e-mail: revista.ambientales@una.ac.cr Ramos-Jiliberto R., Jiliberto-Herrera R.

\section{Discusión}

En este estudio presentamos una metodología y un lenguaje de modelización formal, basados en digrafos signados, útil para sistemas socio-naturales cuya descripción inicial es conceptual. Este lenguaje tiene la ventaja de que solo requiere conocer - o asumir conocer-- el conjunto de variables relevantes del sistema y la red de influencias positivas o negativas entre ellas. En otras palabras, este sistema de modelización no requiere conocer -aunque sí pueden incorporarse- magnitudes de influencia ni ecuaciones que describan relaciones funcionales entre variables, ni valores iniciales de variables; todo ello sí es requisito para la modelización basada en otros lenguajes (p.ej., ecuaciones diferenciales). En síntesis, este tipo de modelización opera con una definición meramente cualitativa del sistema. Lo anterior es una ventaja en consideración a que comúnmente no es posible acceder a información empírica cuantitativa de un sistema complejo. Presentamos también algunas herramientas básicas de análisis de tales sistemas modelizados y de evaluación de escenarios alternativos de intervención basadas en sistemas dinámicos y matrices aleatorias. Adicionalmente, desarrollamos y presentamos nuevas métricas apropiadas para el análisis de sistemas socio-naturales y las proyecciones de acciones de intervención: sensibilidad $S$, reactividad positiva $R$, efectividad $K$ y virtuosismo $U$. Las herramientas de análisis introducidas aquí se basan en la proyección de efectos netos entre las variables de sistema, es decir, sobre la integración de todos los efectos directos e indirectos entre las variables. Esta aproximación obedece, por tanto, a un enfoque sistémico para el abordaje de fenómenos y problemas socio-naturales complejos.

Tomamos como caso de estudio el sistema de sustentabilidad energía-territorio de la región de Coquimbo (Chile). En este estudio de caso, mostramos que es posible identificar escenarios de intervención con buena proyección de éxito basándose en las medidas SRKU propuestas. Sobre la base del manejo de 4 variables de sistema fue posible obtener una proyección con alto porcentaje de efectos deseables y escasa fracción de efectos laterales indeseables. Por supuesto, la metodología presentada permite la evaluación de innumerables escenarios de intervención, con diferente nivel de complejidad, factibilidad de implementación y estructura de costos. Con ello, es posible construir un portafolio de soluciones alternativas con el fin de orientar la toma de decisiones de política pública. Estas orientaciones tienen la característica de estar basadas en herramientas de modelización matemática, replicables y con supuestos que pueden ser validados.

De forma más genérica se ha mostrado que esta modelización constituye una propuesta solvente para formalizar, matemáticamente, una descripción sistémica cualitativa de un sistema socio-natural complejo, propio de los objetos de política pública, en el entendido que aquello modelizado sistémicamente no pretende ser un retrato de una realidad objetiva, sino que presenta la estructura del problema de política pública.

En general hubo una débil correlación entre las medidas de centralidad y las medidas SRKU (Apéndice 6 y 7). De las 24 correlaciones, solo 4 presentaron una magnitud $|\rho| \geq 0.6$, valor que puede considerarse como el umbral inferior de correlaciones fuertes. De estas, la centralidad de cercanía de entrada resultó ser un buen predictor de la sensibilidad $S$, mientras la cercanía de

\begin{tabular}{|c|c|c|}
\hline 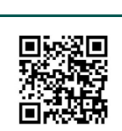 & (c) (i) () () & 15 \\
\hline
\end{tabular}




\section{Revista de CIENCIAS AMBIENTALES Tropical Journal of Environmental Sciences}

Revista de Ciencias Ambientales (Trop J Environ Sci) e-ISSN: 2215-3896 (Enero-Junio, 2021) . Vol 55(1): 1-31 DOI: https://doi.org/10.15359/rca.55-1.1

Open Access: www.revistas.una.ac.cr/ambientales e-mail: revista.ambientales@una.ac.cr Ramos-Jiliberto R., Jiliberto-Herrera R.

salida resulto ser buen predictor de la efectividad $K$. Sin embargo, las medidas más sofisticadas (reactividad positiva $R$ y virtuosismo $U$ ) presentaron, en general, una débil asociación con las medidas de centralidad. Aun así, el punto más fuerte que desfavorece el uso de medidas de centralidad, aun en sus versiones direccionadas (es decir, que respetan para su cálculo la dirección de las influencias), radica en el débil sentido de aplicar medidas de centralidad que no consideran el signo de las influencias ni la valorización de las variables. Una solución factible sería cuantificar por separado las medidas de centralidad para el subgrafo de influencias positivas y para el subgrafo de influencias negativas. Sin embargo, no es obvia ni la interpretación integrada de tal cantidad de medidas ni su utilidad práctica.

En términos matemáticos formales, nuestra metodología se inserta dentro de una clase amplia de procedimientos que se conoce como "análisis estructural de sistemas". Un tipo de análisis relacionado con el nuestro es el propuesto por Godet (1993), denominado metodología MICMAC. Con dicha metodología se analizan, por separado, las influencias directas, de orden 1, e indirectas, de orden $2,3, \ldots, n$, entre las variables del sistema. Las influencias indirectas de orden $k$ se realizan a través de $k$ número de variables intermediarias. Sin embargo, quienes practican la metodología MICMAC - o la teoría prospectiva, dentro de la que se inserta-, suelen centrarse solo en el análisis de las influencias directas (Beinstein, 2016). Con la aproximación MICMAC solo se considera la existencia o inexistencia de influencias, no su signo. También admite utilizar categorías de intensidad de la influencia. La red de influencias, definida de tal modo, se resume en la llamada matriz de análisis estructural, desde la cual se calculan los índices principales para la detección de variables-clave: motricidad y dependencia. Respecto de la presentación sintética de resultados, la metodología MICMAC utiliza ampliamente el llamado plano de motricidad vs. dependencia de las variables. En nuestro caso, representamos una mayor riqueza de resultados, como se expone más abajo. De cualquier modo, la presentación gráfica es solo el punto de partida que facilita la elaboración de una interpretación apropiada de las salidas del modelo. Nuestra aproximación, presentada en este estudio, comparte con MICMAC los propósitos básicos de todo análisis estructural, cual es la descripción y la comprensión del sistema de interés desde un análisis de las relaciones consideradas relevantes entre sus componentes. Por otro lado, nuestra aproximación incorpora una serie de elementos de modelización y análisis de sistemas que no están considerados en MICMAC. Nuestro sistema se representa por un digrafo y su correspondiente matriz de influencias $M$. Sus elementos $m_{\{i j\}}$ incorporan dirección, signo e intensidad de la influencia directa de ${ }_{j}$ sobre ${ }^{x_{i}}$. En contraste, la matriz de análisis estructural usada en MICMAC no considera el signo de la influencia y, por tanto, no distingue entre relaciones de inhibición y de reforzamiento entre variables. A partir de $M$, desarrollamos la matriz de efectos netos $A$. Esta estructura integra todas las vías de influencia directas e indirectas entre pares de variables. Cada uno de sus elementos $a_{i j}$ indica el efecto total de la variable $j$ sobre la variable $i$ del sistema, considerando todo el conjunto de retroalimentaciones y signos implicados en tal relación. Este punto es vital y contrasta fuertemente con MICMAC, donde las vías directas e indirectas se analizan por separado y, por tanto, sumado a que las influencias

\begin{tabular}{|c|c|c|}
\hline 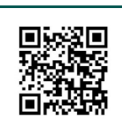 & (c) (i) () () & 16 \\
\hline
\end{tabular}




\section{Revista de CIENCIAS AMBIENTALES Tropical Journal of Environmental Sciences}

Revista de Ciencias Ambientales (Trop J Environ Sci) e-ISSN: 2215-3896 (Enero-Junio, 2021) . Vol 55(1): 1-31 DOI: https://doi.org/10.15359/rca.55-1.1

Open Access: www.revistas.una.ac.cr/ambientales e-mail: revista.ambientales@una.ac.cr Ramos-Jiliberto R., Jiliberto-Herrera R.

carecen de signo, no permiten dar cuenta de posibles cancelaciones entre cadenas paralelas de influencias entre variables. Sobre la matriz $A$ de efectos integrados netos, calculamos las cuatro métricas propuestas en este estudio: $S$ (sensibilidad, cercana a la dependencia de MICMAC), $K$ (efectividad, cercana a la motricidad de MICMAC), $R$ (reactividad positiva, sin análogo en MICMAC) y $U$ (virtuosismo, sin análogo en MICMAC). Las dos métricas más sofisticadas, $R$ y $U$ utilizan la información de la valoración de cada variable, otro aspecto aportante de nuestros métodos. Adicionalmente, nuestra propuesta teórico-metodológica tiene el mérito de avanzar en la definición formal de los elementos que la componen: variables, influencias, efectos netos, digrafo del sistema, matriz de influencias, matriz de efectos netos, escenarios de intervención, métricas descriptivas. Respecto de los escenarios de intervención, nuestra aproximación permite incorporarlos como conjuntos de perturbaciones sostenidas aplicadas directamente sobre los componentes del sistema modelizado.

A pesar de las ventajas ya señaladas atribuibles a este tipo de modelización, esta no puede dejar de ser lo que es: una formalización de un conocimiento cualitativo y parcial de un sistema complejo. Coherente con esto, la forma de modelización aquí presentada no pretende ni constituir una descripción "objetiva" de una realidad ni formular predicciones cuantitativas "duras". Mas bien, esta modelización en el ámbito de las políticas públicas ambientales constituye un avance hacia la sistematización y formalización del conocimiento utilizado para la toma de decisiones. Por supuesto, esta propuesta de modelización tiene sus propias limitaciones, la mayoría de ellas más bien ubicadas en su fase más discursiva que en la de su formalización matemática. En cuanto a la fase discursiva, es pertinente destacar la dificultad propia de arribar a un conjunto limitado de temas claves, elementos que deben integrar la descripción de un objeto de política pública per se complejo. El problema no radica en que ello sea imposible, sino en que se requiere establecer consensos entre un número limitado de actores, lo cual dificulta la realización del ejercicio, en particular al tener en cuenta los escenarios propios en los que se lleva a cabo la formulación de una política pública. Por otro lado, la definición de vinculaciones causales entre las variables, que conduce a la construcción del sistema, requiere pericia, conocimiento y habilidad, que no se adquieren de inmediato. Estas competencias deben ser exhibidas por el personal conductor de los procesos participativos con actores clave que se lleven a efecto para la definición del modelo a utilizar.

En términos de la modelización matemática, también es necesario precisar limitaciones. El método presentado contiene ciertos parámetros libres, que deben ser ajustados con juicio experto: la magnitud de los coeficientes de autolimitación de las variables, la magnitud de las presiones sobre las variables en los escenarios de intervención, la variación en los coeficientes de la matriz de influencias para la simulación computacional, entre otros. Por tanto, hoy no es posible contar con un algoritmo independiente del juicio humano. Esta no es una cualidad exclusiva de esta aproximación, pero sí supone una sistematización y formalización de los pasos de modelización para generar una trazabilidad que permita validar los resultados. Pero, probablemente, la limitación matemática más seria recae en el supuesto de equilibrio del sistema dinámico antes y

\begin{tabular}{|c|c|c|}
\hline 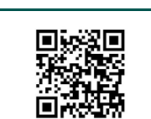 & (c) (1) () (1) & 8 \\
\hline
\end{tabular}




\section{Revista de CIENCIAS AMBIENTALES Tropical Journal of Environmental Sciences}

Revista de Ciencias Ambientales (Trop J Environ Sci) e-ISSN: 2215-3896 (Enero-Junio, 2021) . Vol 55(1): 1-31 DOI: https://doi.org/10.15359/rca.55-1.1

Open Access: www.revistas.una.ac.cr/ambientales e-mail: revista.ambientales@una.ac.cr Ramos-Jiliberto R., Jiliberto-Herrera R.

después de la intervención. A pesar de algunos avances para relajar esta condición (Córdova-Lepe et al., 2012), el supuesto de equilibrio sigue siendo necesario para aplicar este tipo de análisis. Sin embargo, hay evidencia de que, en sistemas naturales que difícilmente presentan un equilibrio estricto, esta metodología es satisfactoria a la luz de la contrastación empírica (Dambacher \& Ramos-Jiliberto, 2007). Recién, el estudio de sistemas dinámicos se ha visto potentemente enriquecido por el desarrollo de la teoría de sistemas dinámicos no-autónomos (Kloeden \& Pötzsche, 2013). Esta teoría ofrece interesantes generalizaciones a los equilibrios puntuales de la aproximación clásica (i.e. sistemas autónomos), por medio de las llamadas "soluciones acotadas enteras". Es de esperar que dichos desarrollos teóricos puedan ser aplicados, en el futuro, al análisis estructural de sistemas socio-naturales.

Todo modelo se aleja, en mayor o menor medida, del sistema que representa. Esta distancia obedece a que los modelos contienen elementos idealizados y, eventualmente, elementos falsos. La idealización comprende la incorporación de un error tolerable, necesario para obtener en el modelo un objeto factible de analizar y entender. Por este motivo, en el proceso de modelización matemática se realizan supuestos orientados a construir un sistema abstracto tan simple como sea posible, pero que en esencia represente el sujeto de interés último. Por otro lado, los supuestos falsos se introducen comúnmente como consecuencia de la insuficiencia de conocimiento acerca del sistema a modelizar. Este tipo de error es menos tolerable, aunque difícil de pesquisar. El riesgo de introducir supuestos falsos es prevalente en el caso de sistemas socio-naturales, frecuentemente asociados a problemáticas contemporáneas de elevada complejidad y a la vez altamente dinámicas. Dado que nuestros modelos están sujetos a incertidumbre en sus supuestos de construcción, resulta verosímil preguntarse qué tan confiables pueden ser nuestras conclusiones derivadas del análisis de tales modelos. En otras palabras, es deseable conducir algún tipo de evaluación de la sensibilidad de nuestros resultados frente a variaciones en los supuestos adoptados en la modelización. La aproximación presentada en este estudio corresponde a un análisis estructural. Es decir, apuntamos a evaluar las consecuencias de la estructura de relaciones entre los elementos incluidos en nuestro sistema modelo. En otras palabras, dada una estructura de un sistema socio-natural, nos preguntamos acerca de qué clase de comportamiento podemos proyectar como consecuencia de determinada intervención sobre este. Lo que debemos evaluar en este sentido, es en qué medida cambian nuestras proyecciones si modificamos los elementos que acompañan la estructura definida del sistema. Estos elementos, por nombrar algunos, son los valores asumidos para las magnitudes de las influencias entre las variables de sistema, las magnitudes asumidas de las presiones que componen los escenarios de intervención, el número de aleatorizaciones utilizado para extraer tendencias, y la medida de tendencia central que utilizamos para caracterizar los resultados. En este estudio, probamos con diferentes niveles de estos valores para detectar eventuales cambios cualitativos de los resultados. En particular, las magnitudes de las influencias fueron variadas aleatoriamente con distribución uniforme, dentro de márgenes razonables. Para efectos de toma de decisión, sería aconsejable extender y documentar profusamente estos análisis de sensibilidad, conducidos tanto mediante la variación

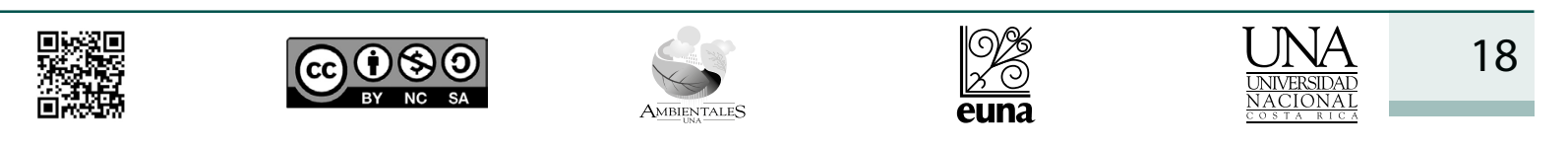




\section{Revista de CIENCIAS AMBIENTALES Tropical Journal of Environmental Sciences}

Revista de Ciencias Ambientales (Trop J Environ Sci) e-ISSN: $2215-3896$ (Enero-Junio, 2021) . Vol 55(1): 1-31 DOI: https://doi.org/10.15359/rca.55-1.1

Open Access: www.revistas.una.ac.cr/ambientales e-mail: revista.ambientales@una.ac.cr Ramos-Jiliberto R., Jiliberto-Herrera R.

libre como sistemática de los valores de los parámetros involucrados en la modelización y en el análisis de escenarios de intervención. Sin embargo, es también razonable preguntarse cuánto cambiarían nuestras proyecciones acerca del comportamiento del sistema, si la estructura de este fuese diferente a la incorporada en el modelo. Esto es, $i$ se mantienen las mismas proyecciones si ciertas influencias entre variables fuesen de signo opuesto, o si algunas influencias fuesen removidas o agregadas? Este enfoque implica permitir variaciones a la estructura del modelo, la que sirve de punto de partida al análisis del sistema, e indagar en las consecuencias de dichas variaciones. Este tipo de análisis de sensibilidad, a cambios en la estructura del modelo no fue abordado en este artículo y bien podría conformar un desarrollo importante en futuros estudios. Por ejemplo, sería adecuado evaluar la sensibilidad de las proyecciones del modelo frente a niveles crecientes de variación aleatoria en el número de entradas nulas de la matriz de influencias, así como a alteraciones aleatorias en el signo de las entradas no nulas de la matriz de influencias. Un enfoque analítico interesante sería otorgar mayor probabilidad de variación a aquellas relaciones con menor evidencia de apoyo. La evaluación de las consecuencias de cambios en la estructura del modelo puede ser relevante en la toma de decisiones socio-naturales. Lo anterior, atendiendo a la elevada dificultad operativa en el establecimiento de un modelo estructural basado en evidencia y, por otro lado, por la pronunciada variabilidad espacial y temporal característica de estos sistemas.

La toma de decisión en política pública y en especial en materias ambientales se beneficia de apoyos basados en un conjunto amplio de argumentos provenientes de evidencia, percepciones, valoraciones, acuerdos, experiencia, historia. En este estudio pretendemos contribuir a la generación de argumentos para la toma de decisiones en políticas públicas ambientales y socio-naturales, Los argumentos que desarrollamos mediante esta aproximación, basada en modelización y análisis de sistemas, son de naturaleza teórica, de proyecciones matemáticas de escenarios plausibles de intervención ejercidos sobre un sistema construido por observaciones humanas. Sostenemos que, para fines de gestión, será provechoso conducir más investigación interdisciplinaria empleando abordajes cuantitativos a modelos cualitativos, posiblemente derivados de teorías y métodos de diversos campos del saber.

\section{Conclusiones}

La aproximación sistémica al estudio de sistemas socio-naturales orientados a la toma de decisiones en política pública exhibe un buen potencial de aplicación. Esto se ve favorecido por los desarrollos teórico-metodológicos que abordamos en este artículo. Los resultados obtenidos para nuestro sistema de estudio sugieren que las medidas de intervención/gestión planificadas en función de las nuevas métricas presentadas en este trabajo, resultan particularmente eficaces en función de los escenarios proyectados.

\begin{tabular}{|c|c|c|}
\hline 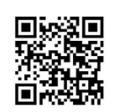 & (c) (1) (9) & 19 \\
\hline
\end{tabular}




\section{Revista de CIENCIAS AMBIENTALES Tropical Journal of Environmental Sciences}

Revista de Ciencias Ambientales (Trop J Environ Sci) e-ISSN: 2215-3896 (Enero-Junio, 2021) . Vol 55(1): 1-31 DOI: https://doi.org/10.15359/rca.55-1.1

Open Access: www.revistas.una.ac.cr/ambientales e-mail: revista.ambientales@una.ac.cr Ramos-Jiliberto R., Jiliberto-Herrera R.

\section{6. Ética y conflicto de intereses}

Los autores declaran que han cumplido totalmente con todos los requisitos éticos y legales pertinentes, tanto durante el estudio como en la producción del manuscrito; que no hay conflictos de intereses de ningún tipo; que todas las fuentes financieras se mencionan completa y claramente en la sección de agradecimientos; y que están totalmente de acuerdo con la versión final editada del artículo.

\section{Agradecimientos}

El trabajo de Rodrigo Ramos-jiliberto fue financiado por el proyecto CONICYT/FONDECYT 1190173. Los autores agradecen a Vanessa Weinberger, y también a la Revista y personas revisoras anónimas por sus sugerencias, que contribuyeron a mejorar este artículo.

\section{Referencias}

Allesina, S., \& Tang, S. (2012). Stability criteria for complex ecosystems. Nature, 483, 205-208. https://doi.org/10.1038/nature10832

Atkinson, G., Dietz, S., Neumayer, E., \& Agarwala, M. (2014). Handbook of Sustainable Development. Second Edition. Edward Elgar Publishing. https://doi.org/10.4337/9781782544708

Barabási, A. L. (2016). Network science. Cambridge University Press.

Beinstein, J. (2016). Manual de prospectiva: Guía para el diseño e implementación de estudios prospectivos. Ministerio de Ciencia, Tecnología e Innovación Productiva.

CMMAD (Comisión Mundial de Medio Ambiente y Desarrollo). (1987). Nuestro futuro común. Alianza Editorial.

Córdova-Lepe, F., Garay-Narváez, L., Ramos-Jiliberto, R., \& Robledo, G. (2012). Sensitivity matrix of an ecological network with periodic dynamics. In R. Mondaini (Ed.), Biomat 2011. International symposium on mathematical and computational biology (73-83). World Scientific: Singapore. https://doi.org/10.1142/9789814397711_0006

Dambacher, J. M., Luh, H. K., Li, H. W., \& Rossignol, P. A. (2003). Qualitative stability and ambiguity in model ecosystems. The American Naturalist, 161(6), 876-888. https://doi. org $/ 10.1086 / 367590$

Dambacher, J. M., Ramos-Jiliberto, R. (2007). Understanding and predicting effects of modified interactions through qualitative analysis of community structure. The Quarterly Review of Biology, 82, 227-50. https://doi.org/10.1086/519966

\begin{tabular}{|c|c|c|}
\hline 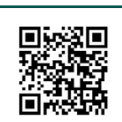 & (c) (i) () (2) & 20 \\
\hline
\end{tabular}




\section{Revista de CIENCIAS AMBIENTALES Tropical Journal of Environmental Sciences}

Revista de Ciencias Ambientales (Trop J Environ Sci) e-ISSN: 2215-3896 (Enero-Junio, 2021) . Vol 55(1): 1-31 DOI: https://doi.org/10.15359/rca.55-1.1 Open Access: www.revistas.una.ac.cr/ambientales e-mail: revista.ambientales@una.ac.cr Ramos-Jiliberto R., Jiliberto-Herrera R.

Deuman S. A., \& Tau Consultora Ambiental S. L. (2017). Construcción de una propuesta de plan energético para la región de Coquimbo. Informe final. Ministerio de Energía, Chile. Santiago de Chile.

Faucheux S., Pearce D., \& Proops J. (1996). Models of Sustainable Development. Edward Elgar Publishing.

Folke, C. (2006). Resilience: The emergence of a perspective for social-ecological systems analyses. Global Environmental Change, 16(3), 253-267. https://doi.org/10.1016/j. gloenvcha.2006.04.002

Gallopin G. (2003). Sostenibilidad y desarrollo sostenible: Un enfoque sistémico. Serie Medio ambiente y desarrollo, CEPAL.

Giampietro M., \& Mayumi K. (1997). A dymamic model of socioeconomic system based on hierarchy theory and its application to sustainability. Structural Change and Economic Dynamics, 8, 453-469. https://doi.org/10.1016/S0954-349X(97)00017-9

Godet, M. (1993). De la anticipación a la acción. Manual de prospectiva y estrategia. Marcombo, Boixareu Eds.

Haydon, D. (1994). Pivotal assumptions determining the relationship between stability and complexity: an analytical synthesis of the stability-complexity debate. The American Naturalist, 144, 14-29. https://doi.org/10.1086/285658

Jiliberto R. (2004). A holarchical model for regional sustainability assessment. Journal of Environmental Assessment Policy and Management, 6(4), 511-538. https://doi.org/10.1142/ S1464333204001833

Jiliberto R. (2018). Ciencia y objetos de política pública, hacia un lenguaje científico posnormal: El caso del cambio climático. En Cuadernos de Beauchef Volúmen I (pp. 106-130) Facultad de Ciencias Físicas y Matemáticas de la Universidad de Chile.

Kloeden, P. E., \& Pötzsche, C. (Eds.) (2014). Nonautonomous Dynamical Systems in the Life Sciences. Springer. https://doi.org/10.2478/msds-2013-0004

Luhmann, N. (2007). La sociedad de la sociedad. México: Herder.

May, R. M. (1972). Will a large complex system be stable? Nature, 238, 413-414. https://doi. org/10.1038/238413a0

Meadows, D. H., Meadows, D. L., Randers, J., \& Behrens, W. W. (1972). Los límites del crecimiento: Informe al Club de Roma sobre el predicamento de la humanidad. Fondo de Cultura Económica.

\begin{tabular}{|c|c|c|c|c|c|}
\hline 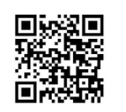 & (c) (1) () (2) & $\underset{\text { AMBENENALIES }}{\infty}$ & $\frac{1 \%}{\text { euna }}$ & 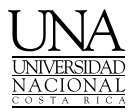 & 21 \\
\hline
\end{tabular}




\section{Revista de CIENCIAS AMBIENTALES Tropical Journal of Environmental Sciences}

Revista de Ciencias Ambientales (Trop J Environ Sci) e-ISSN: 2215-3896 (Enero-Junio, 2021) . Vol 55(1): 1-31 DOI: https://doi.org/10.15359/rca.55-1.1 Open Access: www.revistas.una.ac.cr/ambientales e-mail: revista.ambientales@una.ac.cr Ramos-Jiliberto R., Jiliberto-Herrera R.

Ministerio de Energía. (2018). Guía 2.0 para la Elaboración de Planes Energéticos Regionales. Ministerio Energía, Chile: Santiago de Chile.

Newman, M. (2018). Networks. Inglaterra: Oxford university press. https://doi.org/10.1093/ oso/9780198805090.001.0001

Novak, M., Yeakel, J. D., Noble, A. E., Doak, D. F., Emmerson, M., Estes, J. A., Jacob, U., Tinker, M.T., \& Wootton, J. T. (2016) Characterizing species interactions to understand press perturbations: what is the community matrix? Annual Review of Ecology, Evolution, and Systematics, 47, 409-432. https://doi.org/10.1146/annurev-ecolsys-032416-010215

Parsons, T. (1988). El sistema social. España: Alianza.

Puccia, C. J., \& Levins, R. (1985) Qualitative modeling of complex systems: an introduction to loop analysis and time averaging. Harvard University Press.

Ramos-Jiliberto, R., (2020). Deja a la estructura hablar. Modelización y análisis de sistemas naturales, sociales y socio-ecológicos. Ediciones Universidad Mayor. En prensa.

Sayles, J. S., Garcia, M. M., Hamilton, M., Alexander, S. M., Baggio, J. A., Fischer, A. P., ... \& Pittman, J. (2019). Social-ecological network analysis for sustainability sciences: A systematic review and innovative research agenda for the future. Environmental Research Letters, 14(9), 093003. https://doi.org/10.1088/1748-9326/ab2619

Todorov, V. \& Marinova, D. (2011) Modelling sustainability. Mathematics and Computers in Simulation, 81(7), 1397-1408. https://doi.org/10.1016/j.matcom.2010.05.022

Urquiza Gómez, A., \& Cadenas, H. (2015). Sistemas socio-ecológicos: Elementos teóricos y conceptuales para la discusión en torno a vulnerabilidad hídrica. L'Ordinaire des Amériques, 218. https://doi.org/10.4000/orda.1774

Vázquez, D. P., Ramos-Jiliberto, R., Urbani, P., \& Valdovinos, F. S. (2015). A conceptual framework for studying the strength of plant-animal mutualistic interactions. Ecology letters, 18(4), 385-400. https://doi.org/10.1111/ele.12411

\begin{tabular}{|c|c|c|c|c|}
\hline 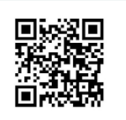 & (c) (i) () () & 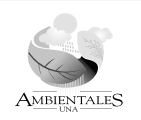 & $\frac{\mid O \%}{2)}$ & 22 \\
\hline
\end{tabular}




\section{Revista de CIENCIAS AMBIENTALES

\section{Apéndices}

Apéndice 1. El digrafo del sistema y su matriz de influencias. Arriba la izquierda, digrafo ponderado de un sistema socioeconómico pequeño, a modo de ejemplo. Nótese que los pesos de las aristas pueden ser positivos o negativos. Arriba a la derecha, la matriz de influencias equivalente. Abajo a la izquierda, ejemplo de intervención (en rojo) aplicada al sistema de estudio. La intervención (I) consiste en una presión negativa (inhibitoria) sobre $x \_2$ y $x \_5$, junto a una presión positiva (reforzadora) sobre $x \_1$. Abajo a la derecha, la matriz de influencias equivalente.

Appendix 1. The digraph of the system and its matrix of influences. Above left, weighted digraph of a small socioeconomic system, as an example. Note that edge weights can be positive or negative. Top right, the equivalent influence matrix. Bottom left, example of intervention (in red) applied to the study system. Intervention (I) consists of a negative (inhibitory) pressure on $x \_2$ and $x \_5$, together with a positive (reinforcing) pressure on $x \_1$. Bottom right, the equivalent influence matrix.

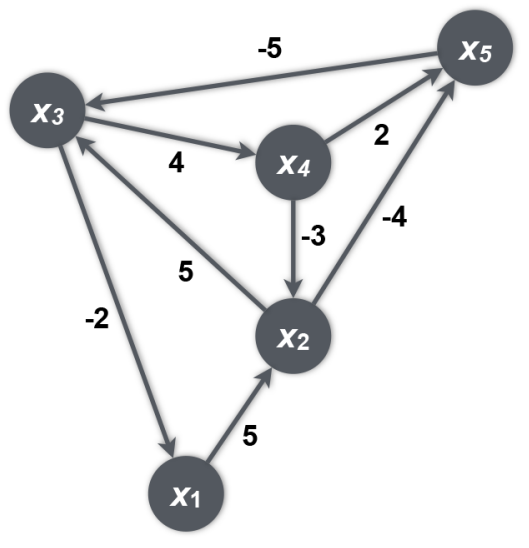

$$
M=\left[\begin{array}{ccccc}
0 & 0 & -2 & 0 & 0 \\
5 & 0 & 0 & -3 & 0 \\
0 & 5 & 0 & 0 & -5 \\
0 & 0 & 4 & 0 & 0 \\
0 & -4 & 0 & 2 & 0
\end{array}\right]
$$

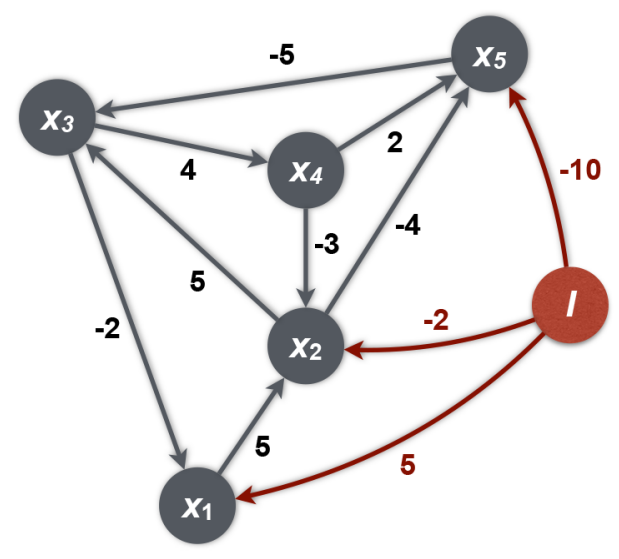

$$
M^{I}=\left[\begin{array}{cccccc}
0 & 0 & -2 & 0 & 0 & 5 \\
5 & 0 & 0 & -3 & 0 & -2 \\
0 & 5 & 0 & 0 & -5 & 0 \\
0 & 0 & 4 & 0 & 0 & 0 \\
0 & -4 & 0 & 2 & 0 & -10 \\
0 & 0 & 0 & 0 & 0 & 0
\end{array}\right]
$$

\begin{tabular}{|c|c|c|c|c|c|}
\hline 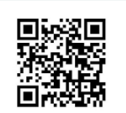 & (c) (i) (5) & $\theta_{\text {AMBIENTALS }}^{6}$ & $\frac{Q \%}{20}$ & $\frac{\text { UNA }}{\frac{\text { UNIVERSIDAD }}{\text { UNACIONAL }}}$ & 23 \\
\hline
\end{tabular}




\section{Revista de CIENCIAS AMBIENTALES Tropical Journal of Environmental Sciences}

Revista de Ciencias Ambientales (Trop J Environ Sci) e-ISSN: 2215-3896 (Enero-Junio, 2021) . Vol 55(1): 1-31 DOI: https://doi.org/10.15359/rca.55-1.1

Open Access: www.revistas.una.ac.cr/ambientales e-mail: revista.ambientales@una.ac.cr Ramos-Jiliberto R., Jiliberto-Herrera R.

Apéndice 2. Elementos del sistema.

Appendix 2. Elements of the system.

Aquí se describen los 20 temas claves identificados, que pasarán a constituirse en los elementos (i.e., variables) de nuestro modelo sistémico.

1. Nivel de energización rural. La región de Coquimbo posee un alto grado de dispersión territorial debido a su geomorfología y la estructura de centros poblados resultantes. Como consecuencia, tiene dificultades para otorgar servicios energéticos a la población de las localidades rurales aisladas. Esto restringe el desarrollo humano y la calidad de vida de esta población.

2. Déficit energético y pobreza rural. En la región existe una relación estrecha entre el déficit de energía y la pobreza rural, en particular en las provincias que presentan problemáticas de escasez hídrica o de aislamiento territorial.

3. Nivel de desconfianza de la comunidad frente a proyectos de energía. Los proyectos energéticos en los últimos años han recibido críticas por parte de la población local, por sus posibles externalidades negativas al medio ambiente. Esta situación se presenta como una tendencia creciente en los últimos años. Si bien en un inicio los conflictos estaban principalmente asociados al posible incremento de material particulado y dióxido de azufre en el aire, la paleta de críticas se ha ampliado. De hecho, todo proyecto energético recibe alguna manifestación crítica o de rechazo. Incluso el desarrollo de las energías renovables genera percepciones críticas.

4. Condicionamiento territorial para el desarrollo energético. Distintos regímenes y situaciones del suelo regional suponen condicionantes para el desarrollo de proyectos energéticos. Entre estas se hayan: áreas en protección oficial de la biodiversidad terrestre, áreas de interés para la biodiversidad terrestre que aún no cuentan con estatus de protección, suelos agrícolas de alta calidad, instrumentos de planificación territorial de nivel comunal, intercomunal y provincial, solicitudes de exploración y explotación minera, existencia de amplias áreas de suelo rural en manos de comunidades agrícolas, alto grado de privatización del suelo regional.

5. Escasez y conflictividad del recurso hídrico. La intensa y prolongada escasez de agua, que posiblemente ha de intensificarse por efecto del cambio climático, ha profundizado los conflictos de uso del recurso hídrico entre sus distintos usuarios: agricultura-minería, minería-energía, varios usuarios-abastecimiento, entre otros. El conflicto por los usos del agua afecta los posibles desarrollos energéticos hidroeléctricos de cualquier escala, así como todo desarrollo energético que requiera del recurso agua. A la vez, desarrollos tendientes a disminuir la escasez hídrica, tales como la desalinización y su elevación a altas cotas, supone

\begin{tabular}{|c|c|c|}
\hline 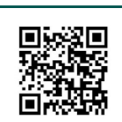 & (c) (1) () (2) (2) & 24 \\
\hline
\end{tabular}




\section{Revista de CIENCIAS AMBIENTALES Tropical Journal of Environmental Sciences}

Revista de Ciencias Ambientales (Trop J Environ Sci) e-ISSN: 2215-3896 (Enero-Junio, 2021) . Vol 55(1): 1-31 DOI: https://doi.org/10.15359/rca.55-1.1

Open Access: www.revistas.una.ac.cr/ambientales e-mail: revista.ambientales@una.ac.cr Ramos-Jiliberto R., Jiliberto-Herrera R.

también una demanda energética relevante a satisfacer, complejizando así la demanda energética regional.

6. Debilidad institucional. La región cuenta solo muy recientemente con una Secretaría Regional Ministerial de Energía, que ha ido sentando bases de una gestión regional del sector, lo que incluye cambios institucionales, establecidos en la Política 2050. Ello permitirá mejorar las coordinaciones al interior del Ministerio de Energía, así como la gobernanza público-privada regional. Sin embargo, históricamente ha existido muy poca coordinación de los actores públicos y privados tanto a nivel regional, como en el nivel del gobierno central. Igualmente, las problemáticas energéticas han estado muy poco presentes en las políticas municipales. En definitiva, y tomando todas las escalas de gobernanza regional, el sector y la política energética han carecido de mecanismos de coordinación eficaces.

7. Dificultades para el desarrollo de las energías renovables. La región presenta un gran potencial, y recientemente un incremento, de la generación eléctrica en base a energías renovables, principalmente en proyectos eólicos y en menor medida solares. No obstante, pese a que las oportunidades de negocios y desafíos energéticos son significativas, existen fuertes barreras e incertidumbres que inhiben la inversión de energías renovables. Estas barreras son normativas, financieras, técnicas, ambientales, sociales, e incluso combinaciones entre varias de estas. También se constatan barreras institucionales, relacionadas a emisión de permisos, solicitudes de diverso tipo, entre otros.

8. Descapitalización del mundo rural. Existen una serie de problemáticas en los modelos institucionales y de negocios que se manifiestan en barreras para la generación de oportunidades energéticas rurales. El problema del acceso a la energía en ámbitos rurales es recurrente, los modelos institucionales no han cambiado en este sentido, y no han sido capaces de enfrentar el problema de cobertura en aquellas zonas donde los modelos de negocios de las empresas distribuidoras no son rentables. Allí el problema persiste, a pesar de intentos de electrificación rural. Estos obstáculos impiden el desarrollo de iniciativas energéticas, como son los pequeños medios de generación distribuidos. Se observa potencial para estas iniciativas, las que además favorecerían el necesario recambio productivo, desde actividades hasta ahora agrícolas hacia actividades de generación y distribución de energía. El acceso a la financiación es, sin duda, otro elemento relevante, como lo son también los problemas de naturaleza más técnica como la limitada disponibilidad de líneas de distribución, lo que también supone un factor de coste relevante. Un papel similar juega el desconocimiento y debilidades de información en asuntos energéticos en general y, en particular, la carencia de capacidades técnicas para el desarrollo de proyectos de energías renovables.

9. Riesgos ambientales de las energías renovables. Los proyectos eólicos y solares, pero en particular los últimos, suelen requerir una extensa área de intervención. Esto lleva implícitos

\begin{tabular}{|c|c|c|}
\hline 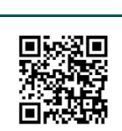 & (c) (1) ()(2) & 25 \\
\hline
\end{tabular}




\section{Revista de CIENCIAS AMBIENTALES Tropical Journal of Environmental Sciences}

Revista de Ciencias Ambientales (Trop J Environ Sci) e-ISSN: 2215-3896 (Enero-Junio, 2021) . Vol 55(1): 1-31 DOI: https://doi.org/10.15359/rca.55-1.1

Open Access: www.revistas.una.ac.cr/ambientales e-mail: revista.ambientales@una.ac.cr Ramos-Jiliberto R., Jiliberto-Herrera R.

ciertos riesgos, hasta ahora poco considerados, de afección directa y sinérgica o acumulativa sobre la flora y fauna en estado de conservación, o de valores paisajísticos o arqueológicos.

10. Nivel de gestión de cuencas hidrográficas. La región se encuentra en una situación problemática frente a los recursos hídricos. Un reflejo de esto es el estado de la gestión y planificación de cuencas hidrográficas que presenta un bajo nivel de integración, pese a contar con un plan maestro para la gestión de recursos hídricos en la región, alineado recientemente a la política Nacional de Gestión Integrada de Cuencas.

11. Sequía y desertificación. La región de Coquimbo afronta problemas serios derivados de los procesos de desertificación y sequía. Esta situación ha llevado a la declaración de escasez hídrica para todas las comunas de la región desde ya hace varios años, las que se renuevan sistemáticamente. La última situación de escasez se establece mediante el Decreto del Ministerio de Obras Públicas n. ${ }^{\circ} 72$ de 01.07.2020. Actualmente la zona con mayores problemas de erosión en el país es la región de Coquimbo, con el 84 \% de sus suelos erosionados, $65.3 \%$ de ellos en categorías severa o muy severa, lo que significa 2.9 millones de hectáreas con riesgo de erosión actual.

12. Nivel de insatisfacción frente a procesos participativos. Entre los actores regionales involucrados en los procesos participativos existe un consenso respecto de la limitada efectividad de la participación en el ámbito decisional de las políticas públicas. Existe un descrédito y una falta de credibilidad sobre los procesos participativos, y se identifica la necesidad de formalizar y asegurar procesos efectivos de participación y seguimiento.

13. Despoblamiento rural. La región sufre un proceso de mutaciones en la ruralización. Por un lado, aunque menos intenso que el que se verifica a nivel nacional, se reproduce una desruralización y, por otro lado, diversificado según cuenca y en ciertos valles, se manifiesta un acelerado proceso de urbanización en zonas rurales. Este proceso de cambios debilita la estructura territorial regional, restándole riqueza y resiliencia, reduciendo los servicios territoriales a escala regional.

14. Concentración de la población en conurbación Serena-Coquimbo. La conurbación Serena-Coquimbo se ha transformado en el principal atractor de población en la región, es destino de importantes flujos demográficos intrarregionales e interregionales. Esto ha transformado y seguirá transformando, de forma significativa, la estructura del complejo de centros poblados regionales, y ha generado un sistema muy descompensado con un gran polo urbano y un conjunto, escasamente integrado, de centros poblados menores.

15. Nivel de desintegración funcional del territorio. El territorio regional presenta, gracias a sus condiciones topográficas, un escaso nivel de integración funcional entre sus principales

\begin{tabular}{|c|c|c|c|c|c|}
\hline 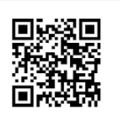 & (c) (1) (9) & 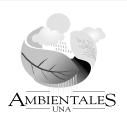 & $\frac{O \%}{2)}$ & 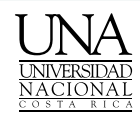 & 26 \\
\hline
\end{tabular}




\section{Revista de
CIENCIAS AMBIENTALES Tropical Journal of Environmental Sciences}

Revista de Ciencias Ambientales (Trop J Environ Sci) e-ISSN: 2215-3896 (Enero-Junio, 2021) . Vol 55(1): 1-31 DOI: https://doi.org/10.15359/rca.55-1.1

Open Access: www.revistas.una.ac.cr/ambientales e-mail: revista.ambientales@una.ac.cr Ramos-Jiliberto R., Jiliberto-Herrera R.

elementos estructurantes, su sistema de ciudades, sus sistemas de comunicación, sus actividades económicas y usos del suelo, entre otros.

16. Monoproductividad de economías territoriales. La escasa integración territorial regional ha dado lugar o se ha asociado a una fuerte especialización productiva. Esto ha generado subterritorios caracterizados por una fuerte especialización económica, con bajo grado de diversificación interna y con una escasa integración entre estas economías.

17. Desarticulación económica y territorial minera. La minería se ha transformado en un gran colonizador del territorio regional, formándolo y transformándolo de manera intensa. No obstante, su carácter de economía de enclave ha limitado notablemente su capacidad para diversificar las economías subregionales y locales, o para mejorar la estructura y resiliencia de la estructura territorial regional. Por el contrario, la minería ha tendido a simplificar las economías locales y subregionales.

18. Autonomía territorial minera. El desarrollo minero, en particular el de gran escala, constituye una de las fuerzas motrices más relevantes de modificación del territorio regional. Su desarrollo, no obstante, no es condicionado ni se funda en la estructura regional existente, sino que más bien genera sus propias lógicas y estructuras territoriales, muchas de las cuales tienen un alcance regional. Esto genera una disfuncionalidad básica entre la estructura territorial regional y el desarrollo territorial minero, con fuertes desequilibrios.

19. Concentración territorial de la infraestructura energética. La infraestructura de trasmisión y distribución, así como la de generación, se distribuyen de forma muy desigual en la región, muy concentrada en torno a la Ruta 5, principal arteria de transporte terrestre del país, y las pocas líneas de trasmisión hacia las actividades mineras del interior.

20. Concentración territorial de la demanda. La demanda energética regional se encuentra extremadamente concentrada, por un lado, en el ámbito urbano en la conurbación Serena-Coquimbo y, por otro, en el ámbito industrial en la actividad minera en dos o tres localizaciones específicas. Ambas modalidades, suponen en conjunto, casi la totalidad del consumo de energía.

\begin{tabular}{|c|c|c|c|c|c|}
\hline 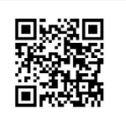 & (c) (1) (5) () & $\underbrace{}_{\text {AMEIENTIISS }}$ & $\frac{O \%}{2)}$ & $\frac{\text { UNA }}{\frac{\text { UNIVERSIDAD }}{\text { NACIONAL }}}$ & 27 \\
\hline
\end{tabular}




\section{Revista de CIENCIAS AMBIENTALES \\ Soste 1980 Tropical Journal of Environmental Sciences}

Revista de Ciencias Ambientales (Trop J Environ Sci) e-ISSN: 2215-3896 (Enero-Junio, 2021) . Vol 55(1): 1-31 DOI: https://doi.org/10.15359/rca.55-1.1 Open Access: www.revistas.una.ac.cr/ambientales e-mail: revista.ambientales@una.ac.cr Ramos-Jiliberto R., Jiliberto-Herrera R.

Apéndice 3. Métricas propuestas para el análisis prospectivo de sistemas socio-naturales complejos. Appendix 3. Proposed metrics for the prospective analysis of complex socio-natural systems.

Sensibilidad. $S_{i} \in[0,1]$ indica la propensión de la variable $x_{i}$ a ser afectada por presiones ejercidas sobre otras variables del sistema.

$$
S_{i}=\frac{\sum_{j \neq i}\left|a_{i j}\right|}{2(n-1)}
$$

Efectividad. $K_{i} \in[0,1]$ indica la capacidad de la variable $x_{i}$ para ejercer cambios en los valores de equilibrio de las $\neq$ variables del sistema.

$$
K_{j=} \frac{\sum_{i \neq j}\left|a_{i j}\right|}{2(n-1)}
$$

Virtuosismo. $U_{j} \in[-1,1]$ indica la capacidad de la variable $x_{j}$ de transmitir cambios valorados positivamente en las otras variables del sistema. Es decir, es la capacidad de la variable $x_{j}$ de, dado un aumento en ella, aumentar el valor de las variables positivas y disminuir el valor de las variables negativas.

$$
U_{j}=\frac{\sum_{i \neq \mathrm{j}} v_{i} a_{i j}}{\sum_{i \neq \mathrm{j}}\left|a_{i j}\right|}
$$

Reactividad positiva. $R_{i} \in[-1,1]$ indica la propensión de la variable $x_{i}$ a ser incrementada por cambios valorados positivamente en otras variables del sistema o reducida por cambios valorados negativamente.

$$
R_{i}=\frac{\sum_{j \neq i} v_{j} a_{i j}}{\sum_{j \neq i}\left|a_{i j}\right|}
$$

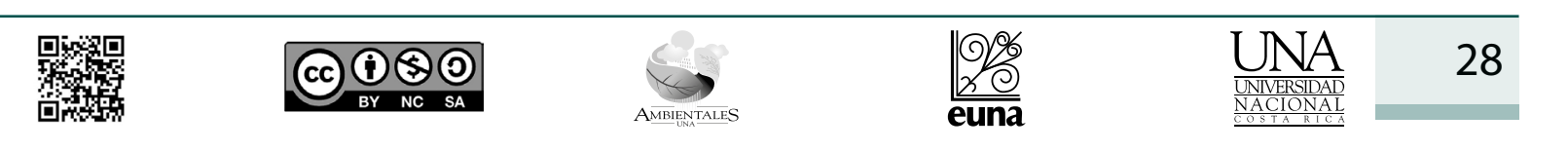




\section{Revista de CIENCIAS AMBIENTALES Tropical Journal of Environmental Sciences}

Revista de Ciencias Ambientales (Trop J Environ Sci) e-ISSN: 2215-3896 (Enero-Junio, 2021) . Vol 55(1): 1-31 DOI: https://doi.org/10.15359/rca.55-1.1 Open Access: www.revistas.una.ac.cr/ambientales e-mail: revista.ambientales@una.ac.cr Ramos-Jiliberto R., Jiliberto-Herrera R.

Apéndice 4. Detalle de valores de propiedades SRKU de las variables de sistema (Var), previo a la intervención (S, $R, K, U)$, posterior a la intervención en el escenario 1 (S-I1, etc.) y posterior a la intervención en el escenario 2 (S-I2, etc.). En la segunda columna se muestra la valoración (positiva=1 o negativa=-1) de cada variable.

Appendix 4. Detail of SRKU property values of the system variables (Var), prior to the intervention (S, $R, K, U)$, after the intervention in scenario 1 (S-I1, etc.) and after the intervention on stage 2 (S-I2, etc.). The second column shows the evaluation (positive $=1$ or negative $=-1$ ) of each variable.

\begin{tabular}{cccccccccccccc}
\hline Var & Valor & $\mathbf{S}$ & $\mathbf{R}$ & $\mathbf{K}$ & $\mathbf{U}$ & $\mathbf{S}-\mathbf{I 1}$ & $\mathbf{R}-\mathbf{I 1}$ & $\mathbf{K}-\mathbf{I 1}$ & $\mathbf{U}-\mathbf{I 1}$ & $\mathbf{S}-\mathbf{I 2}$ & $\mathbf{R}-\mathbf{I} 2$ & K-I2 & U-I2 \\
\hline 1 & 1 & 0.342 & 0.846 & 0.263 & 0.800 & 0.342 & 0.692 & 0.263 & 0.800 & 0.342 & 0.846 & 0.263 & 0.800 \\
2 & -1 & 0.342 & -0.077 & 0.342 & -0.538 & 0.421 & 0.000 & 0.368 & -0.429 & 0.342 & -0.077 & 0.342 & -0.538 \\
3 & -1 & 0.184 & -0.429 & 0.184 & 0.714 & 0.184 & -0.714 & 0.263 & 0.800 & 0.263 & -0.200 & 0.211 & 0.750 \\
4 & -1 & 0.132 & 0.600 & 0.316 & 0.500 & 0.184 & 0.143 & 0.395 & 0.600 & 0.132 & 0.600 & 0.263 & 0.400 \\
5 & -1 & 0.237 & 0.333 & 0.395 & -0.067 & 0.368 & 0.571 & 0.395 & -0.067 & 0.237 & 0.333 & 0.421 & -0.125 \\
6 & -1 & 0.421 & 0.750 & 0.447 & -0.647 & 0.421 & 0.750 & 0.474 & -0.667 & 0.421 & 0.750 & 0.421 & -0.875 \\
7 & -1 & 0.447 & -0.765 & 0.316 & 0.833 & 0.447 & -0.765 & 0.368 & 0.857 & 0.447 & -0.765 & 0.368 & 0.857 \\
8 & -1 & 0.474 & -0.222 & 0.158 & 0.667 & 0.474 & -0.222 & 0.237 & 0.778 & 0.474 & -0.222 & 0.211 & 0.750 \\
9 & -1 & 0.132 & 0.600 & 0.237 & 0.556 & 0.132 & 0.600 & 0.395 & 0.600 & 0.237 & 0.556 & 0.237 & 0.556 \\
10 & 1 & 0.316 & -0.833 & 0.368 & 0.143 & 0.316 & -0.833 & 0.316 & 0.167 & 0.289 & -0.818 & 0.368 & 0.143 \\
11 & -1 & 0.158 & 0.333 & 0.342 & -0.077 & 0.158 & 0.333 & 0.316 & 0.000 & 0.158 & 0.333 & 0.368 & 0.000 \\
12 & -1 & 0.316 & 0.833 & 0.079 & 0.333 & 0.316 & 0.833 & 0.105 & 0.000 & 0.368 & 0.714 & 0.079 & 0.333 \\
13 & -1 & 0.395 & 0.200 & 0.289 & -0.455 & 0.474 & 0.333 & 0.289 & -0.455 & 0.368 & 0.143 & 0.289 & -0.455 \\
14 & -1 & 0.289 & -0.091 & 0.342 & -0.077 & 0.342 & 0.231 & 0.368 & 0.000 & 0.289 & -0.091 & 0.342 & -0.077 \\
15 & -1 & 0.368 & -0.714 & 0.263 & 0.000 & 0.474 & -0.333 & 0.263 & 0.000 & 0.368 & -0.714 & 0.263 & 0.000 \\
16 & -1 & 0.395 & -0.333 & 0.342 & 0.231 & 0.474 & -0.111 & 0.368 & 0.286 & 0.368 & -0.429 & 0.342 & 0.231 \\
17 & -1 & 0.289 & -0.273 & 0.211 & -0.250 & 0.342 & -0.077 & 0.211 & -0.250 & 0.289 & -0.273 & 0.211 & -0.250 \\
18 & -1 & 0.316 & 0.167 & 0.316 & -0.167 & 0.342 & 0.231 & 0.342 & -0.077 & 0.316 & 0.167 & 0.316 & -0.167 \\
19 & -1 & 0.000 & 0.000 & 0.316 & -0.167 & 0.000 & 0.000 & 0.368 & 0.000 & 0.000 & 0.000 & 0.289 & -0.273 \\
20 & -1 & 0.316 & -0.333 & 0.342 & 0.077 & 0.289 & -0.455 & 0.395 & 0.067 & 0.289 & -0.273 & 0.395 & 0.200 \\
\hline & & & & & & & & & & & & &
\end{tabular}

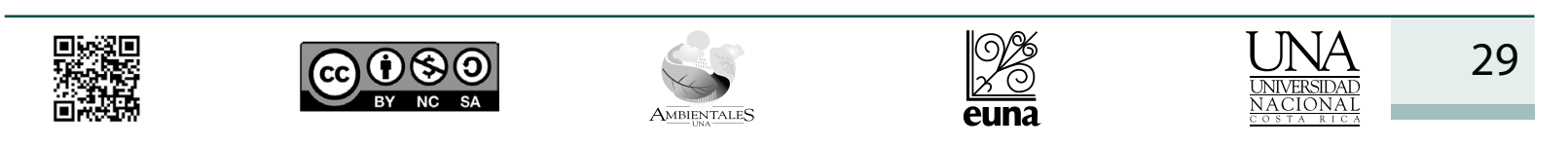




\section{Revista de CIENCIAS AMBIENTALES \\ $\longrightarrow \begin{gathered}\text { Desde } \\ \text { Since } 1980\end{gathered}$ Tropical Journal of Environmental Sciences}

Revista de Ciencias Ambientales (Trop J Environ Sci) e-ISSN: 2215-3896

(Enero-Junio, 2021) . Vol 55(1): 1-31 DOI: https://doi.org/10.15359/rca.55-1.1

Open Access: www.revistas.una.ac.cr/ambientales e-mail: revista.ambientales@una.ac.cr Ramos-Jiliberto R., Jiliberto-Herrera R.

Apéndice 5. Valores de centralidad direccionada para las variables de sistema (Var). Grado total (Grado_tot), grado de entrada (Grado_in), grado de salida (Grado_out), intermediación (Interm), cercanía de entrada (Cerc_in) y cercanía de salida (Cerc_out).

Appendix 5. Addressed centrality values for system variables (Var). Total grade (Grade_tot), entry grade (Grade_in), exit grade (Grade_out), intermediation (Interm), entry closeness (Cerc_in) and exit closeness (Cerc_out).

\begin{tabular}{ccccccc}
\hline Var & Grado_tot & Grado_in & Grado_out & Interm & Cerc_in & Cerc_out \\
\hline 1 & 2 & 1 & 1 & 6.750 & 0.014 & 0.012 \\
2 & 5 & 2 & 3 & 72.500 & 0.017 & 0.015 \\
3 & 5 & 4 & 1 & 27.833 & 0.014 & 0.012 \\
4 & 3 & 1 & 2 & 22.083 & 0.010 & 0.013 \\
5 & 6 & 2 & 4 & 63.250 & 0.012 & 0.020 \\
6 & 4 & 1 & 3 & 173.250 & 0.018 & 0.020 \\
7 & 6 & 5 & 1 & 174.250 & 0.025 & 0.015 \\
8 & 3 & 2 & 1 & 37.000 & 0.019 & 0.012 \\
9 & 3 & 1 & 2 & 1.000 & 0.009 & 0.013 \\
10 & 3 & 1 & 2 & 73.000 & 0.014 & 0.017 \\
11 & 3 & 1 & 2 & 1.750 & 0.012 & 0.017 \\
12 & 2 & 1 & 1 & 13.500 & 0.014 & 0.010 \\
13 & 4 & 2 & 2 & 42.000 & 0.019 & 0.013 \\
14 & 3 & 1 & 2 & 14.500 & 0.014 & 0.015 \\
15 & 7 & 4 & 3 & 48.250 & 0.018 & 0.014 \\
16 & 7 & 4 & 3 & 59.500 & 0.022 & 0.015 \\
17 & 3 & 2 & 1 & 7.917 & 0.016 & 0.011 \\
18 & 4 & 2 & 2 & 27.500 & 0.017 & 0.014 \\
19 & 1 & 0 & 1 & 0.000 & 0.000 & 0.014 \\
20 & 6 & 3 & 3 & 61.167 & 0.014 & 0.018 \\
\hline
\end{tabular}

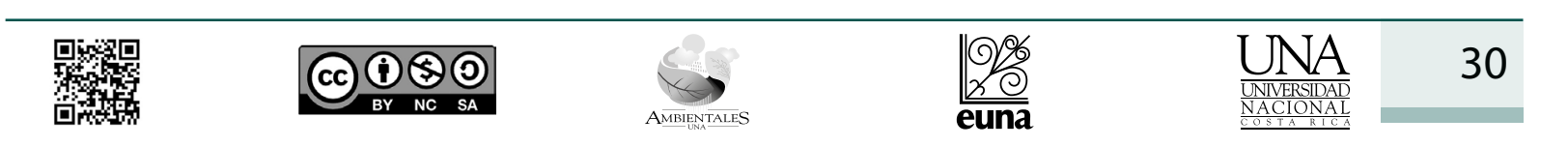




\section{Revista de CIENCIAS AMBIENTALES \\ $\longrightarrow$ Desde 1980 Tropical Journal of Environmental Sciences}

Revista de Ciencias Ambientales (Trop J Environ Sci) e-ISSN: 2215-3896 (Enero-Junio, 2021) . Vol 55(1): 1-31 DOI: https://doi.org/10.15359/rca.55-1.1 Open Access: www.revistas.una.ac.cr/ambientales e-mail: revista.ambientales@una.ac.cr Ramos-Jiliberto R., Jiliberto-Herrera R.

Apéndice 6. Valores del coeficiente de correlación lineal para las 24 combinaciones de medidas de centralidad y SRKU.

Appendix 6. Values of the linear correlation coefficient for the 24 combinations of centrality measures and SRKU.

\begin{tabular}{ccccccc}
\hline & Grado_tot & Grado_in & Grado_out & Interm & Cerc_in & Cerc_out \\
\hline S & 0.434 & 0.471 & 0.125 & 0.582 & 0.907 & 0.033 \\
R & -0.502 & -0.642 & -0.004 & -0.260 & -0.364 & -0.044 \\
K & 0.299 & -0.072 & 0.664 & 0.492 & 0.014 & 0.888 \\
U & -0.046 & 0.288 & -0.506 & -0.127 & 0.068 & -0.435 \\
\hline
\end{tabular}

Apéndice 7. Significación estadística (valor-P) para la correlación entre las 24 combinaciones de medidas de centralidad $y$ SRKU.

Appendix 7. Statistical significance ( $P$-value) for the correlation between the 24 combinations of centrality measures and SRKU.

\begin{tabular}{lllllll}
\hline & Grado_tot & Grado_in & Grado_out & Interm & Cerc_in & Cerc_out \\
\hline S & 0.056 & 0.036 & 0.600 & 0.007 & 0.000 & 0.890 \\
R & 0.024 & 0.002 & 0.988 & 0.269 & 0.115 & 0.855 \\
K & 0.200 & 0.763 & 0.001 & 0.028 & 0.954 & 0.000 \\
U & 0.847 & 0.219 & 0.023 & 0.593 & 0.775 & 0.055 \\
\hline
\end{tabular}

